\title{
Numerical boundary feedback stabilisation of non-uniform hyperbolic systems of balance laws
}

\author{
Mapundi K. Banda ${ }^{a}$ and Gediyon Y. Weldegiyorgis ${ }^{\mathrm{a}}$ \\ ${ }^{a}$ Department of Mathematics and Applied Mathematics, University of Pretoria, Lynnwood \\ Rd, Hatfield, Pretoria, 0002, South Africa.
}

\author{
ARTICLE HISTORY \\ Compiled August 3, 2018
}

\begin{abstract}
In this paper, numerical boundary stabilisation of a non-uniform hyperbolic system of balance laws is studied. For the numerical discretisation of the balance laws, a first order explicit upwind scheme is used for the spatial discretisation; and for the temporal discretisation a splitting technique is employed. A discrete $L^{2}$-Lyapunov function is employed to investigate conditions for the stability of the system. After constructing discrete numerical Lyapunov functionals, we prove an asymptotic exponential stability result for a class of non-uniform linear hyperbolic systems of balance laws. Convergence of the solution to its equilibrium is proved. Further application of the approach to practical problems through concrete examples is presented together with suggestions for numerical implementation. The numerical computations also demonstrate the stability of the numerical scheme with parameters chosen to satisfy the stability requirements.
\end{abstract}

\section{KEYWORDS}

Conservation laws, Systems of non-uniform balance laws, Controllability, Feedback Stabilisation, Lyapunov Functions

\section{Introduction}

In this paper, numerical stabilisation of a hyperbolic $k \times k$ system of non-uniform balance laws in one-space dimension by boundary controls is considered. Hyperbolic systems of balance laws are useful in describing the transport of a set of physical quantities that have a mathematical physics and an engineering interest. Therefore, these kinds of systems have a large number of applications in the physical modelling of different phenomena. Some of the relevant examples include the telegrapher equation that describes the propagation of an electric signal along an electric line, Karaguler (2008); the Aw-Rascle and Greenberg equation that describes the dynamics of road traffic flow, Siebel \& Mauser (2006); the Saint-Venant equation that describes the dynamics of shallow water flow along an open channel, Bastin, Coron, \& d'Andréa Novel (2008); the Saint-Venant Exner equation that describes the dynamics of shallow water flow with sediment transportation along an open channel, A. Diagne, Bastin, \& Coron (2012); and the Euler equations for gas dynamics that describe the behaviour of gas flow through a medium Chalons, Girardin, \& Kokh (2012). In addition, hyperbolic 
systems of balance laws are used to model networked flow in which case the flow through the edge of the network is governed by the hyperbolic balance law. The flow through the node (or vertex) of the network, outgoing or ingoing to the edge, is coupled by algebraic conditions motivated by physical considerations Bastin et al. (2008).

The mathematical analysis of hyperbolic balance laws has been an active field of research for more than fifty years Dafermos (2009). For more details of the mathematical properties of hyperbolic balance laws, the reader is referred to the introduction part in Dafermos (2009). In particular, in the recent past the boundary control problem of hyperbolic systems of balance laws has been a very active area of research Bastin \& Coron (2011); Coron, Bastin, \& d'Andréa Novel (2008); Coron, d'Andréa Novel, \& Bastin (2007); A. Diagne et al. (2012); Di Meglio, Vazquez, \& Krstic (2013); Dos Santos, Bastin, Coron, \& d'Andréa Novel (2008); Gugat \& Herty (2011); Litrico \& Fromion (2009); Prieur (2009); Prieur, Winkin, \& Bastin (2008); Vazquez, Coron, Krstic, \& Bastin (2011).

In such cases, researchers have focused on well-posedness (i.e. existence, uniqueness and stability) of a hyperbolic system of balance laws Coron (2007); Gugat, Herty, Klar, Leugering, \& Schleper (2012). Besides the existence and uniqueness, a stability analysis for boundary control of a hyperbolic system of balance laws has been studied for different categories such as for a linear hyperbolic system of conservation laws in Litrico \& Fromion (2009), a linear hyperbolic system of balance laws in Prieur et al. (2008), a non-linear hyperbolic system of conservation laws in Prieur (2009) and networks of such in de Halleux, Prieur, Coron, d'Andréa Novel, \& Bastin (2003).

In general, the objective is to stabilize a solution of a hyperbolic system of balance laws to a preferred equilibrium state by finding boundary control action that will be used at the boundary points (or at the nodes of the network in networked flow). For the stability analysis of a boundary control of a hyperbolic system of balance laws, there exists a candidate Lyapunov function which is a positive quadratic form. According to the Lyapunov stability analysis theorem, the time derivative of the Lyapunov function needs to be in a negative quadratic form to exhibit the stability of the system. This technique was introduced for a linear hyperbolic system of conservation laws in Coron et al. (2007); Dos Santos et al. (2008), a linear hyperbolic system of balance laws in Bastin, Coron, \& d'Andréa Novel (2009) and later for networks of hyperbolic $2 \times 2$ systems of balance laws in Bastin et al. (2008). This approach was briefly analysed for a linear hyperbolic system of balance laws in A. Diagne et al. (2012). Using the discussion of the linear case, this approach was further extended to a non-linear hyperbolic system of conservation laws in Coron et al. (2008).

For practical purposes, numerical aspects of a hyperbolic system of balance laws have also undergone tremendous development Prieur, Girard, \& Witrant $(2012,2014)$. For instance, in the above mentioned literature and in general, numerical results for the stability analysis of a hyperbolic system of balance laws have been given to support the theoretical stability analysis. This stability analysis has been applied to some important examples such as shallow water flow along an open channel Bastin et al. (2008); Coron et al. (2007); de Halleux \& Bastin (2002); A. Diagne et al. (2012) and gas dynamics Banda, Herty, \& Klar (2006). However, there is limited literature on the numerical boundary stability analysis of hyperbolic systems of balance laws. Recently, researchers have investigated conditions for the numerical stability of a discretised linear hyperbolic system of conservation laws by considering a single flow domain Banda \& Herty (2013) and networked flow domain Dick et al. (2014). In these studies, a discrete Lyapunov function was introduced and the decay of the discrete Lyapunov proved. The purpose of the current work is to consider a numerical boundary sta- 
bilisation of a non-uniform linear hyperbolic system of balance laws. The analysis of a numerical discretisation of stabilisation problems with boundary controls for such a linear hyperbolic system of balance laws will be undertaken. In particular the numerical analysis of the convergence of a numerical solution of a non-uniform linear hyperbolic system of balance laws to a preferred equilibrium is proved. An investigation of conditions for the decay of discrete solutions of non-uniform linear hyperbolic systems of balance laws will be presented. The numerical boundary stabilisation will also be applied to relevant examples in the field of fluid dynamics.

For details of numerical analysis the reader is referred to Section 3. The numerical stability analysis technique used in this work is similar to that used in Banda \& Herty (2013) and Gottlich \& Schillen (2017). A discrete Lyapunov function will be applied in the investigation of conditions for decay rates depending on numerical schemes that have been employed. Numerical results for this study will be presented in Section 4 .

\section{Boundary Stabilisation of Linear Hyperbolic System of Balance Laws}

In this section, we consider the following non-uniform linear hyperbolic $k \times k$ system of balance laws in one-space dimension:

$$
\partial_{t} W+\Lambda(x) \partial_{x} W+\Pi(x) W=0, \quad x \in[0, l], \quad t \in[0,+\infty),
$$

where $W:=\left[w_{1}, \ldots, w_{k}\right]^{T}:[0, l] \times[0,+\infty) \rightarrow \Omega$, a non-empty, open and connected subset of $\mathbb{R}^{k}, \Lambda(x):=\operatorname{diag}\left\{\lambda_{1}(x), \ldots, \lambda_{k}(x)\right\}$, and $\Pi(x)$ is a real $k \times k$ matrix. Here we assume that $\Lambda(x)$ has non-zero real smooth diagonal entries that can be arranged as follows

$$
\lambda_{k}(x) \leq \cdots \leq \lambda_{m+1}(x)<0<\lambda_{m}(x) \leq \cdots \leq \lambda_{1}(x), \quad \forall x \in[0, l] .
$$

In addition, we introduce the following notation

$$
\begin{aligned}
& W:=\left[W^{+}, W^{-}\right]^{T}, \quad \Lambda(x):=\operatorname{diag}\left\{\Lambda^{+}(x),-\Lambda^{-}(x)\right\} \\
& \text { and }|\Lambda(x)|:=\operatorname{diag}\left\{\Lambda^{+}(x), \Lambda^{-}(x)\right\},
\end{aligned}
$$

with $W^{+}:=\left[w_{1}, \ldots, w_{m}\right]^{T}, W^{-}:=\left[w_{m+1}, \ldots, w_{k}\right]^{T}$

$$
\Lambda^{+}(x):=\operatorname{diag}\left\{\lambda_{1}(x), \ldots, \lambda_{m}(x)\right\} \text { and } \Lambda^{-}(x):=\operatorname{diag}\left\{\left|\lambda_{m+1}(x)\right|, \ldots,\left|\lambda_{k}(x)\right|\right\} .
$$

Thus, the system (1) with this notation can be expressed as follows:

$\partial_{t}\left[\begin{array}{l}W^{+} \\ W^{-}\end{array}\right]+\left[\begin{array}{cc}\Lambda^{+}(x) & 0 \\ 0 & -\Lambda^{-}(x)\end{array}\right] \partial_{x}\left[\begin{array}{l}W^{+} \\ W^{-}\end{array}\right]+\Pi(x)\left[\begin{array}{l}W^{+} \\ W^{-}\end{array}\right]=0, x \in[0, l], t \in[0,+\infty)$.

In order to formulate a Cauchy problem associated with the linear system (2), we 
prescribe the following initial conditions

$$
\left[\begin{array}{l}
W^{+}(x, 0) \\
W^{-}(x, 0)
\end{array}\right]:=\left[\begin{array}{l}
W_{0}^{+}(x) \\
W_{0}^{-}(x)
\end{array}\right], \quad x \in(0, l),
$$

where $\left[W_{0}^{+}, W_{0}^{-}\right]^{T}:[0, l] \rightarrow \Omega$ and boundary conditions of the form

$$
\left[\begin{array}{l}
W^{+}(0, t) \\
W^{-}(l, t)
\end{array}\right]:=\underbrace{\left[\begin{array}{ll}
K_{11} & K_{12} \\
K_{21} & K_{22}
\end{array}\right]}_{K}\left[\begin{array}{l}
W^{+}(l, t) \\
W^{-}(0, t)
\end{array}\right], \quad t>0 .
$$

At this point, it can also be pointed out that since

$$
\partial_{x}(\Lambda(x) W)=\Lambda(x) \partial_{x} W+\Lambda^{\prime}(x) W
$$

the system (2) can be written in the following form

$$
\partial_{t} W+\partial_{x}(\Lambda(x) W)+\left(\Pi(x)-\Lambda^{\prime}(x)\right) W=0 .
$$

Consideration for the initial boundary compatibility condition of the form

$$
\left[\begin{array}{l}
W^{+}(0,0) \\
W^{-}(l, 0)
\end{array}\right]:=K\left[\begin{array}{l}
W^{+}(l, 0) \\
W^{-}(0,0)
\end{array}\right]
$$

is made and the fact that it satisfies the existence and uniqueness of a solution to the Cauchy problem (2), (3), (4) is stated in Bastin \& Coron (2016). Further, a weak formulation of a solution to the Cauchy problem (2), (3), (4) is also defined in Bastin \& Coron (2016).

The central theme of this paper is to analyse a numerical stability for the nonuniform linear hyperbolic system of balance laws. To prepare the setting, we discuss the analytical stability analysis for this system. At first, we give the following definition of exponential stability of the linear system.

Definition 2.1 (Exponential Stability Bastin \& Coron (2016)). The linear system (2) with boundary condition (4) is exponentially stable for the $L^{2}$-norm if there exist $\eta>0$ and $C>0$ such that, for every initial condition $W_{0}(x) \in L^{2}\left((0, l) ; \mathbb{R}^{k}\right)$, the $L^{2}$-solution to the Cauchy problem (2), (3), (4) satisfies

$$
\|W(\cdot, t)\|_{L^{2}\left((0, l) ; \mathbb{R}^{k}\right)} \leq C e^{-\eta t}\left\|W_{0}\right\|_{L^{2}\left((0, l) ; \mathbb{R}^{k}\right)}, \quad \forall t>0 .
$$

In order to stabilise the linear system (2) with boundary condition (4) a candidate $L^{2}$-Lyapunov function is used in Bastin \& Coron (2016) and it is defined as follows:

$$
\mathcal{L}(t):=\int_{0}^{l} W^{T} \Phi(x) W d x, \quad t \geq 0
$$


where $\Phi(x):=\operatorname{diag}\left\{P^{+} e^{-\mu x}, P^{-} e^{\mu x}\right\}$ with $P^{+}:=\operatorname{diag}\left\{p_{1}, \ldots, p_{m}\right\}$ and $P^{-}:=$ $\operatorname{diag}\left\{p_{m+1}, \ldots, p_{k}\right\}$, where $p_{i}>0, i=1, \ldots, k$.

Using the Lyapunov function in (7), we have the following result:

Theorem 2.2 (Bastin \& Coron (2016)). The system (2) with boundary condition (4) is exponentially stable for the $L^{2}-$ norm if there exists $\mu>0$ and $p_{i}>0, i=1, \ldots, k$, such that the following matrices

$$
\left[\begin{array}{cc}
\Lambda^{+}(l) P^{+} e^{-\mu l} & 0 \\
0 & \Lambda^{-}(0) P^{-}
\end{array}\right]-K^{T}\left[\begin{array}{cc}
\Lambda^{+}(0) P^{+} & 0 \\
0 & \Lambda^{-}(l) P^{-} e^{\mu l}
\end{array}\right] K
$$

and

$$
\mu|\Lambda(x)| \Phi(x)-\Lambda^{\prime}(x) \Phi(x)+\Pi^{T}(x) \Phi(x)+\Phi(x) \Pi(x), \quad \forall x \in[0, l]
$$

are positive definite quadratic forms.

Remark 1. From Theorem 2.2, one sees that a matrix $K$ is determined by positive constants $\mu>0$ and $p_{i}>0, i=1, \ldots, k$, which is discussed in Section 4.

A special case of the system (2), which is a linear system with constant coefficients, can be expressed as follows:

$$
\partial_{t}\left[\begin{array}{l}
W^{+} \\
W^{-}
\end{array}\right]+\left[\begin{array}{cc}
\Lambda^{+} & 0 \\
0 & -\Lambda^{-}
\end{array}\right] \partial_{x}\left[\begin{array}{l}
W^{+} \\
W^{-}
\end{array}\right]+\Pi\left[\begin{array}{l}
W^{+} \\
W^{-}
\end{array}\right]=0, \quad x \in[0, l], \quad t \in[0,+\infty),
$$

where $\Lambda^{+}:=\operatorname{diag}\left\{\lambda_{1}, \ldots, \lambda_{m}\right\}, \Lambda^{-}:=\operatorname{diag}\left\{\left|\lambda_{m+1}\right|, \ldots,\left|\lambda_{k}\right|\right\}$ and $\Pi$ is a real $k \times k$ constant matrix. Then, the result in Theorem 2.2 for the system (10) with boundary condition (4) is stated as follows:

Corollary 2.3 (A. Diagne et al. (2012)). The system (10) with boundary condition (4) is exponentially stable for the $L^{2}$-norm if there exist $\mu>0$ and $p_{i}>0, i=1,2, \ldots, k$, such that the following matrices

$$
\left[\begin{array}{cc}
\Lambda^{+} P^{+} e^{-\mu l} & 0 \\
0 & \Lambda^{-} P^{-}
\end{array}\right]-K^{T}\left[\begin{array}{cc}
P^{+} \Lambda^{+} & 0 \\
0 & P^{-} e^{\mu l} \Lambda^{-}
\end{array}\right] K
$$

and

$$
\mu|\Lambda| \Phi(x)+\Pi^{T} \Phi(x)+\Phi(x) \Pi, \quad \forall x \in[0, l]
$$

are positive definite quadratic forms.

One of the requirements in Corollary 2.3 is to show that the matrix (11) is positive definite, which is alternatively described in the following remark.

Remark 2. As stated in Bastin \& Coron (2016), if there exists $\mu>0$ and $p_{i}>$ $0, i=1, \ldots, k$ such that the matrix (12) is positive definite and $\left\|\Delta K \Delta^{-1}\right\|<1$, with $\Delta:=\sqrt{P|\Lambda|}$, where $P:=\operatorname{diag}\left\{P^{+}, P^{-}\right\}$, then the system in (10) with boundary condition (4) is exponentially stable for the $L^{2}-$ norm. 
With this background, the formulation of the discrete numerical boundary stabilisation can be presented. The conditions under which a numerical stabilisation is asymptotically stable will be derived and proofs presented to back such claims in the next section, Section 3 .

\section{Numerical Boundary Stabilisation of Non-uniform Linear Hyperbolic System of Balance Laws}

In this section, we investigate conditions for a numerical stability analysis of one dimensional non-uniform linear hyperbolic system of balance laws. Thus, we consider the Cauchy problem (2), (3), (4) where the independent variables, $x$ and $t$, are defined on finite intervals $[0, l]$ and $[0, T]$, respectively, with $l>0$ and $T>0$. In order to obtain a numerical solution for this system, the finite volume methods (FVM) LeVeque (2002) will be used. For simplicity, we use a uniform grid with grid size for the temporal and spatial intervals denoted by $\Delta t$ and $\Delta x$, respectively. Hence, there are a finite number of grid points along the time and the space directions, which are denoted by $t^{n}:=n \Delta t, n=0, \ldots, N$ and $x_{j-\frac{1}{2}}=j \Delta x, j=0, \ldots, J$, with $N \Delta t=T$ and $J \Delta x=l$, respectively. Moreover, the left and right boundary points are denoted by $x_{-\frac{1}{2}}$ and $x_{J-\frac{1}{2}}$, respectively. Let $x_{j}=\left(j+\frac{1}{2}\right) \Delta x$ for $j=0, \ldots, J-1$ denote cell centres.

The next step in FVM is to approximate the cell average of $W$ over each cell $\left(x_{j-\frac{1}{2}}, x_{j+\frac{1}{2}}\right)$. Hence, the value $W_{j}^{n}$ approximates the $j$ th cell average at time $t^{n}, n=$ $0, \ldots, N$ and it can be expressed as

$$
W_{j}^{n}=\frac{1}{\Delta x} \int_{x_{j-\frac{1}{2}}}^{x_{j+\frac{1}{2}}} W\left(x, t^{n}\right) d x, \quad j=0, \ldots, J-1 .
$$

Since $W$ is a smooth function, the approximate value, $W_{j}^{n}$, can be used to approximate the value of $W$ at the cell-centre in a numerical quadrature. In this case the midpoint value of $W$ in each cell is approximated by $W_{j}^{n}$ at each midpoint of the cells. Thus the midpoint quadrature rule is applied for the integral over the spatial domain $[0, l]$ and will be approximated by $\sum_{j=0}^{J-1} W_{j}^{n} \Delta x$ at each time step $t^{n}, n=0, \ldots, N$.

By integrating the system (5) over $\left[x_{j-\frac{1}{2}}, x_{j+\frac{1}{2}}\right) \times\left[t^{n}, t^{n+1}\right)$ and applying first order upwind explicit scheme with a time splitting method, the following discretisation of the Cauchy problem (2), (3), (4) for $n=0, \ldots, N-1$ is introduced:

$$
\begin{aligned}
& {\left[\begin{array}{c}
\widetilde{W}^{+}{ }_{j} \\
\widetilde{W^{-}} \\
j
\end{array}\right]=\left[\begin{array}{c}
W_{j}^{+n} \\
W_{j}^{-n}
\end{array}\right]-\frac{\Delta t}{\Delta x}\left[\begin{array}{cc}
\Lambda_{j-1}^{+} & 0 \\
0 & \Lambda_{j+1}^{-}
\end{array}\right]\left[\begin{array}{l}
W_{j}^{+n}-W_{j-1}^{+n} \\
W_{j}^{-n}-W_{j+1}^{-n}
\end{array}\right], n=0, \ldots, N-1} \\
& {\left[\begin{array}{c}
W_{j}^{+n+1} \\
W_{j}^{-n+1}
\end{array}\right]=\left[\begin{array}{c}
\widetilde{W^{+}}{ }_{j}^{n} \\
\widetilde{W^{-}}{ }_{j}
\end{array}\right]-\Delta t \Pi_{j}\left[\begin{array}{c}
\widetilde{W^{+}}{ }^{n} \\
{\widetilde{W^{-}}}_{j}^{n}
\end{array}\right], j=0, \ldots, J-1 ;}
\end{aligned}
$$




$$
\begin{aligned}
& W_{j}^{0}=\frac{1}{\Delta x} \int_{x_{j-\frac{1}{2}}}^{x_{j+\frac{1}{2}}} W_{0}(x) d x, \quad j=0, \ldots, J-1 ; \\
& {\left[\begin{array}{c}
W_{-1}^{+n+1} \\
W_{J}^{-n+1}
\end{array}\right]=K\left[\begin{array}{l}
W_{J-1}^{+n+1} \\
W_{0}^{-n+1}
\end{array}\right], \quad n=0, \ldots, N-1 .}
\end{aligned}
$$

We now define exponential stability of the discretised Cauchy problem (14) as follows:

Definition 3.1. The discretised non-uniform linear hyperbolic system (14a) with the discretised boundary conditions (14c) is exponentially stable if there exist $\eta>0$ and $C>0$ such that, for every $W_{j}^{0} \in L^{2}\left(\left(x_{j-\frac{1}{2}}, x_{j+\frac{1}{2}}\right) ; \mathbb{R}^{k}\right), j=0, \ldots, J-1$, the solution to the discretised Cauchy problem (14) satisfies

$$
\Delta x \sum_{j=0}^{J-1}\left[W_{j}^{n}\right]^{T}\left[W_{j}^{n}\right] \leq C e^{-\eta t^{n}} \Delta x \sum_{j=0}^{J-1}\left[W_{j}^{0}\right]^{T}\left[W_{j}^{0}\right], \quad n=0, \ldots, N .
$$

In order to obtain conditions for the stability of the Cauchy problem, we define the following discrete Lyapunov function

$$
\mathcal{L}^{n}=\Delta x \sum_{j=0}^{J-1}\left(W_{j}^{n}\right)^{T} \Phi_{j} W_{j}^{n}, \quad n=0, \ldots, N,
$$

with $\Phi_{j}:=\operatorname{diag}\left\{P^{+} e^{-\mu x_{j}}, P^{-} e^{\mu x_{j}}\right\}$ where $P^{+}:=\operatorname{diag}\left\{p_{1}, \ldots, p_{m}\right\}$ and $P^{-}:=$ $\operatorname{diag}\left\{p_{m+1}, \ldots, p_{k}\right\}$ with $p_{i}>0, \quad i=1, \ldots, k$.

With the above background material, the main result of the paper can now be stated and proved as follows:

Theorem 3.2. Let $T>0$ be fixed and for each $j=0, \ldots, J-1$ assume $\lambda_{i, j}>0, i=1, \ldots, m$ and $\lambda_{i, j}<0, i=m+1, \ldots, k$. Let the discrete Lyapunov function be defined by (15). If the CFL condition $\frac{\Delta t}{\Delta x} \max _{\substack{1 \leq i \leq k \\ 0 \leq j \leq J-1}}\left|\lambda_{i, j}\right| \leq 1$ holds and there exists real $\mu>0, p_{i}>0, i=1, \ldots, k, \alpha=\min _{\substack{1 \leq i \leq k \\ 0 \leq j \leq J-1}}\left|\lambda_{i, j}\right|$ and $\beta=\max _{\substack{1 \leq i \leq k \\ 0 \leq j \leq J-1}} \lambda_{i, j}^{\prime}$, such that $0<\mu \alpha e^{-\mu \Delta x}-\beta<1$,

$$
\Pi_{j}^{T} \Phi_{j}+\Phi_{j} \Pi_{j}-\Delta t \Pi_{j}^{T} \Phi_{j} \Pi_{j}, \quad j=0, \ldots, J-1, \quad \text { is positive semi-definite }
$$

and

$$
\left[\begin{array}{cc}
P^{+} e^{-\mu x_{J}} \Lambda_{J-1}^{+} & 0 \\
0 & P^{-} e^{\mu x_{-1}} \Lambda_{0}^{-}
\end{array}\right]-K^{T}\left[\begin{array}{cc}
P^{+} e^{-\mu x_{0}} \Lambda_{-1}^{+} & 0 \\
0 & P^{-} e^{\mu x_{J-1}} \Lambda_{J}^{-}
\end{array}\right] K,
$$

is positive definite, then the numerical solution $W_{j}^{n}$ of the Cauchy problem (14) converges to 0 for the $L^{2}$-norm.

Proof. The time derivative of the Lyapunov function (7) is approximated by using 
the discrete Lyapunov function (15), which is obtained in the following form

$$
\frac{\mathcal{L}^{n+1}-\mathcal{L}^{n}}{\Delta t}=\frac{\mathcal{L}^{n+1}-\widetilde{\mathcal{L}}^{n}}{\Delta t}+\frac{\widetilde{\mathcal{L}}^{n}-\mathcal{L}^{n}}{\Delta t}, \quad n=0, \ldots, N,
$$

where

$$
\widetilde{\mathcal{L}}^{n}:=\Delta x \sum_{j=0}^{J-1}\left(\widetilde{W}_{j}^{n}\right)^{T} \Phi_{j} \widetilde{W}_{j}^{n}
$$

In order to show that the time derivative (18) is a negative definite quadratic form, it suffices to show that both approximations of the time derivatives

$$
\frac{\mathcal{L}^{n+1}-\widetilde{\mathcal{L}}^{n}}{\Delta t}=\frac{\Delta x}{\Delta t} \sum_{j=0}^{J-1}\left[\left(W_{j}^{n+1}\right)^{T} \Phi_{j} W_{j}^{n+1}-\left(\widetilde{W}_{j}^{n}\right)^{T} \Phi_{j} \widetilde{W}_{j}^{n}\right],
$$

and

$$
\frac{\widetilde{\mathcal{L}}^{n}-\mathcal{L}^{n}}{\Delta t}=\frac{\Delta x}{\Delta t} \sum_{j=0}^{J-1}\left[\left(\widetilde{W}_{j}^{n}\right)^{T} \Phi_{j} \widetilde{W}_{j}^{n}-\left(W_{j}^{n}\right)^{T} \Phi_{j} W_{j}^{n}\right]
$$

are negative definite quadratic forms.

We use now the discretised system (14a) with boundary condition (14c) to obtain the following

$$
\begin{aligned}
\frac{\mathcal{L}^{n+1}-\widetilde{\mathcal{L}}^{n}}{\Delta t} & =\frac{\Delta x}{\Delta t} \sum_{j=0}^{J-1}\left[\left(\widetilde{W}_{j}^{n}-\Delta t \Pi_{j} \widetilde{W}_{j}^{n}\right)^{T} \Phi_{j}\left(\widetilde{W}_{j}^{n}-\Delta t \Pi_{j} \widetilde{W}_{j}^{n}\right)-\left(\widetilde{W}_{j}^{n}\right)^{T} \Phi_{j}\left(\widetilde{W}_{j}^{n}\right)\right], \\
& =\frac{\Delta x}{\Delta t} \sum_{j=0}^{J-1}\left[\left(\widetilde{W}_{j}^{n}\right)^{T} \Phi_{j}\left(\widetilde{W}_{j}^{n}\right)-\left(\widetilde{W}_{j}^{n}\right)^{T} \Phi_{j}\left(\widetilde{W}_{j}^{n}\right)\right] \\
& -\Delta x \sum_{j=0}^{J-1}\left[\left(\widetilde{W}_{j}^{n}\right)^{T} \Pi_{j}^{T} \Phi_{j}\left(\widetilde{W}_{j}^{n}\right)+\left(\widetilde{W}_{j}^{n}\right)^{T} \Phi_{j} \Pi_{j}\left(\widetilde{W}_{j}^{n}\right)-\Delta t\left(\widetilde{W}_{j}^{n}\right)^{T} \Pi_{j}^{T} \Phi_{j} \Pi_{j}\left(\widetilde{W}_{j}^{n}\right)\right], \\
& =-\Delta x \sum_{j=0}^{J-1}\left(\widetilde{W}_{j}^{n}\right)^{T}\left[\Pi_{j}^{T} \Phi_{j}+\Phi_{j} \Pi_{j}-\Delta t \Pi_{j}^{T} \Phi_{j} \Pi_{j}\right]\left(\widetilde{W}_{j}^{n}\right),
\end{aligned}
$$


and

$$
\begin{aligned}
\frac{\widetilde{\mathcal{L}}^{n}-\mathcal{L}^{n}}{\Delta t}=\frac{\Delta x}{\Delta t} \sum_{j=0}^{J-1} & {\left[\left(W_{j}^{n}-\frac{\Delta t}{\Delta x}\left[\begin{array}{cc}
\Lambda_{j-1}^{+} & 0 \\
0 & \Lambda_{j+1}^{-}
\end{array}\right]\left[\begin{array}{l}
W_{j}^{+n}-W_{j-1}^{+n} \\
W_{j}^{-n}-W_{j+1}^{-n}
\end{array}\right]\right)\right)^{T} \Phi_{j} } \\
& \left.\left(W_{j}^{n}-\frac{\Delta t}{\Delta x}\left[\begin{array}{cc}
\Lambda_{j-1}^{+} & 0 \\
0 & \Lambda_{j+1}^{-}
\end{array}\right]\left[\begin{array}{l}
W_{j}^{+n}-W_{j-1}^{+n} \\
W_{j}^{-n}-W_{j+1}^{-n}
\end{array}\right]\right)-\left(W_{j}^{n}\right)^{T} \Phi_{j}\left(W_{j}^{n}\right)\right], \\
= & \frac{\Delta x}{\Delta t} \sum_{j=0}^{J-1}\left[\left(W_{j}^{n}-D_{j}\left(W_{j}^{n}-\Xi_{j}^{n}\right)\right)^{T} \Phi_{j}\left(W_{j}^{n}-D_{j}\left(W_{j}^{n}-\Xi_{j}^{n}\right)\right)-\left(W_{j}^{n}\right)^{T} \Phi_{j}\left(W_{j}^{n}\right)\right],
\end{aligned}
$$

with $D_{j}:=\left[\begin{array}{cc}D_{j-1}^{+} & 0 \\ 0 & D_{j+1}^{-}\end{array}\right]$and $\Xi_{j}^{n}:=\left[\begin{array}{c}W_{j-1}^{+n} \\ W_{j+1}^{-n}\end{array}\right]$, where $D_{j-1}^{+}:=\frac{\Delta t}{\Delta x} \Lambda_{j-1}^{+}$and $D_{j+1}^{-}:=$ $\frac{\Delta t}{\Delta x} \Lambda_{j+1}^{-}$. Then,

$$
\begin{aligned}
\frac{\widetilde{\mathcal{L}}^{n}-\mathcal{L}^{n}}{\Delta t}=\frac{\Delta x}{\Delta t} \sum_{j=0}^{J-1}[ & \left(W_{j}^{n}\right)^{T} \Phi_{j}\left(W_{j}^{n}\right)-2\left(W_{j}^{n}\right)^{T} \Phi_{j} D_{j}\left(W_{j}^{n}\right)+2\left(W_{j}^{n}\right)^{T} \Phi_{j} D_{j}\left(\Xi_{j}^{n}\right) \\
& +\left(W_{j}^{n}\right)^{T} D_{j} \Phi_{j} D_{j}\left(W_{j}^{n}\right)+\left(\Xi_{j}^{n}\right)^{T} D_{j} \Phi_{j} D_{j}\left(\Xi_{j}^{n}\right) \\
& \left.-2\left(W_{j}^{n}\right)^{T} D_{j} \Phi_{j} D_{j}\left(\Xi_{j}^{n}\right)-\left(W_{j}^{n}\right)^{T} \Phi_{j}\left(W_{j}^{n}\right)\right], \\
=\frac{\Delta x}{\Delta t} \sum_{j=0}^{J-1}[ & -2\left(W_{j}^{n}\right)^{T} \Phi_{j} D_{j}\left(W_{j}^{n}\right)+2\left(W_{j}^{n}\right)^{T}\left(I-D_{j}\right) \Phi_{j} D_{j}\left(\Xi_{j}^{n}\right) \\
& \left.+\left(W_{j}^{n}\right)^{T} D_{j} \Phi_{j} D_{j}\left(W_{j}^{n}\right)+\left(\Xi_{j}^{n}\right)^{T} D_{j} \Phi_{j} D_{j}\left(\Xi_{j}^{n}\right)\right] .
\end{aligned}
$$

By the CFL condition and since $\left(I-D_{j}\right) \Phi_{j} D_{j}$ is a positive definite diagonal matrix, we have $I-D_{j} \geq 0$ and using Young's inequality, we obtain

$$
\begin{aligned}
2\left(W_{j}^{n}\right)^{T}\left(I-D_{j}\right) \Phi_{j} D_{j}\left(\Xi_{j}^{n}\right) \leq & \left(W_{j}^{n}\right)^{T}\left(I-D_{j}\right) \Phi_{j} D_{j}\left(W_{j}^{n}\right)+\left(\Xi_{j}^{n}\right)^{T}\left(I-D_{j}\right) \Phi_{j} D_{j}\left(\Xi_{j}^{n}\right), \\
= & \left(W_{j}^{n}\right)^{T} \Phi_{j} D_{j}\left(W_{j}^{n}\right)-\left(W_{j}^{n}\right)^{T} D_{j} \Phi_{j} D_{j}\left(W_{j}^{n}\right) \\
& +\left(\Xi_{j}^{n}\right)^{T} \Phi_{j} D_{j}\left(\Xi_{j}^{n}\right)-\left(\Xi_{j}^{n}\right)^{T} D_{j} \Phi_{j} D_{j}\left(\Xi_{j}^{n}\right) .
\end{aligned}
$$

Therefore,

$$
\begin{aligned}
\frac{\widetilde{\mathcal{L}}^{n}-\mathcal{L}^{n}}{\Delta t} \leq & \frac{\Delta x}{\Delta t} \sum_{j=0}^{J-1}\left[-\left(W_{j}^{n}\right)^{T} \Phi_{j} D_{j}\left(W_{j}^{n}\right)+\left(\Xi_{j}^{n}\right)^{T} \Phi_{j} D_{j}\left(\Xi_{j}^{n}\right)\right], \\
= & -\sum_{j=0}^{J-1}\left[\begin{array}{c}
W_{j}^{+n} \\
W_{j}^{-n}
\end{array}\right]^{T} \Phi_{j}\left[\begin{array}{cc}
\Lambda_{j-1}^{+} & 0 \\
0 & \Lambda_{j+1}^{-}
\end{array}\right]\left[\begin{array}{c}
W^{+n} \\
j \\
W_{j}^{-n}
\end{array}\right] \\
& +\sum_{j=0}^{J-1}\left[\begin{array}{c}
W_{j-1}^{+n} \\
W_{j+1}^{-n}
\end{array}\right]^{T} \Phi_{j}\left[\begin{array}{cc}
\Lambda_{j-1}^{+} & 0 \\
0 & \Lambda_{j+1}^{-}
\end{array}\right]\left[\begin{array}{c}
W_{j-1}^{+n} \\
W_{j+1}^{-n}
\end{array}\right] .
\end{aligned}
$$


Consider the second term on the right hand side of the above equation (22) complemented by $x_{j}=x_{j-1}+\Delta x$ and $x_{j}=x_{j+1}-\Delta x$ :

$$
\begin{aligned}
& \sum_{j=0}^{J-1}\left[\begin{array}{c}
W_{j-1}^{+n} \\
W_{j+1}^{-n}
\end{array}\right]^{T} \Phi_{j}\left[\begin{array}{cc}
\Lambda_{j-1}^{+} & 0 \\
0 & \Lambda_{j+1}^{-}
\end{array}\right]\left[\begin{array}{l}
W_{j-1}^{+n} \\
W_{j+1}^{-n}
\end{array}\right] \\
& =\sum_{j=0}^{J-1}\left[\begin{array}{c}
W_{j-1}^{+n} \\
W_{j+1}^{-n}
\end{array}\right]^{T}\left[\begin{array}{cc}
P^{+} e^{-\mu x_{j}} \Lambda_{j-1}^{+} & 0 \\
0 & P^{-} e^{\mu x_{j}} \Lambda_{j+1}^{-}
\end{array}\right]\left[\begin{array}{c}
W_{j-1}^{+n} \\
W_{j+1}^{-n}
\end{array}\right], \\
& =\sum_{j=0}^{J-1}\left[\begin{array}{l}
W_{j-1}^{+n} \\
W_{j+1}^{-n}
\end{array}\right]^{T}\left[\begin{array}{cc}
e^{-\mu \Delta x} P^{+} e^{-\mu x_{j-1}} \Lambda_{j-1}^{+} & 0 \\
0 & e^{-\mu \Delta x} P^{-} e^{\mu x_{j+1}} \Lambda_{j+1}^{-}
\end{array}\right]\left[\begin{array}{c}
W_{j-1}^{+n} \\
W_{j+1}^{-n}
\end{array}\right] \text {, } \\
& =e^{-\mu \Delta x} \sum_{j=0}^{J-1}\left[\begin{array}{c}
W_{j}^{+n} \\
W_{j}^{-n}
\end{array}\right]^{T} \Phi_{j}\left[\begin{array}{cc}
\Lambda_{j}^{+} & 0 \\
0 & \Lambda_{j}^{-}
\end{array}\right]\left[\begin{array}{c}
W_{j}^{+n} \\
W_{j}^{-n}
\end{array}\right] \\
& +\left[\begin{array}{c}
W_{-1}^{+n} \\
W_{J}^{-n}
\end{array}\right]^{T}\left[\begin{array}{cc}
P^{+} e^{-\mu x_{0}} \Lambda_{-1}^{+} & 0 \\
0 & P^{-} e^{\mu x_{J-1}} \Lambda_{J}^{-}
\end{array}\right]\left[\begin{array}{c}
W_{-1}^{+n} \\
W_{J}^{-n}
\end{array}\right] \\
& -\left[\begin{array}{c}
W_{J-1}^{+n} \\
W_{0}^{-n}
\end{array}\right]^{T}\left[\begin{array}{cc}
P^{+} e^{-\mu x_{J}} \Lambda_{J-1}^{+} & 0 \\
0 & P^{-} e^{\mu x_{-1}} \Lambda_{0}^{-}
\end{array}\right]\left[\begin{array}{c}
W_{J-1}^{+n} \\
W_{0}^{-n}
\end{array}\right] \text {, } \\
& =e^{-\mu \Delta x} \sum_{j=0}^{J-1}\left[\begin{array}{c}
W_{j}^{+n} \\
W_{j}^{-n}
\end{array}\right]^{T} \Phi_{j}\left[\begin{array}{cc}
\Lambda_{j}^{+} & 0 \\
0 & \Lambda_{j}^{-}
\end{array}\right]\left[\begin{array}{c}
W_{j}^{+n} \\
W_{j}^{-n}
\end{array}\right] \\
& +\left[\begin{array}{c}
W_{J-1}^{+n} \\
W_{0}^{-n}
\end{array}\right]^{T} K^{T}\left[\begin{array}{cc}
P^{+} e^{-\mu x_{0}} \Lambda_{-1}^{+} & 0 \\
0 & P^{-} e^{\mu x_{J-1}} \Lambda_{J}^{-}
\end{array}\right] K\left[\begin{array}{c}
W_{J-1}^{+n} \\
W_{0}^{-n}
\end{array}\right] \\
& -\left[\begin{array}{c}
W_{J-1}^{+n} \\
W_{0}^{-n}
\end{array}\right]^{T}\left[\begin{array}{cc}
P^{+} e^{-\mu x_{J}} \Lambda_{J-1}^{+} & 0 \\
0 & P^{-} e^{\mu x_{-1}} \Lambda_{0}^{-}
\end{array}\right]\left[\begin{array}{c}
W_{J-1}^{+n} \\
W_{0}^{-n}
\end{array}\right] \text {. }
\end{aligned}
$$

By using assumption (17), we have

$$
\begin{aligned}
\sum_{j=0}^{J-1}\left[\begin{array}{c}
W_{j-1}^{+n} \\
W_{j+1}^{-n}
\end{array}\right]^{T} & \Phi_{j}\left[\begin{array}{cc}
\Lambda_{j-1}^{+} & 0 \\
0 & \Lambda_{j+1}^{-}
\end{array}\right]\left[\begin{array}{l}
W_{j-1}^{+n} \\
W_{j+1}^{-n}
\end{array}\right] \\
& \leq e^{-\mu \Delta x} \sum_{j=0}^{J-1}\left[\begin{array}{l}
W_{j}^{+n} \\
W_{j}^{-n}
\end{array}\right]^{T} \Phi_{j}\left[\begin{array}{cc}
\Lambda_{j}^{+} & 0 \\
0 & \Lambda_{j}^{-}
\end{array}\right]\left[\begin{array}{c}
W_{j}^{+n} \\
W_{j}^{-n}
\end{array}\right] .
\end{aligned}
$$


Therefore,

$$
\begin{aligned}
& \frac{\widetilde{\mathcal{L}}^{n}-\mathcal{L}^{n}}{\Delta t} \leq-\sum_{j=0}^{J-1}\left[\begin{array}{c}
W_{j}^{+n} \\
W_{j}^{-n}
\end{array}\right]^{T} \Phi_{j}\left[\begin{array}{cc}
\Lambda_{j-1}^{+} & 0 \\
0 & \Lambda_{j+1}^{-}
\end{array}\right]\left[\begin{array}{c}
W_{j}^{+n} \\
W_{j}^{-n}
\end{array}\right] \\
& +e^{-\mu \Delta x} \sum_{j=0}^{J-1}\left[\begin{array}{c}
W_{j}^{+n} \\
W_{j}^{-n}
\end{array}\right]^{T} \Phi_{j}\left[\begin{array}{cc}
\Lambda_{j}^{+} & 0 \\
0 & \Lambda_{j}^{-}
\end{array}\right]\left[\begin{array}{l}
W_{j}^{+n} \\
W_{j}^{-n}
\end{array}\right], \\
& =\sum_{j=0}^{J-1}\left[\begin{array}{c}
W_{j}^{+n} \\
W_{j}^{-n}
\end{array}\right]^{T} \Phi_{j}\left[\begin{array}{cc}
e^{-\mu \Delta x} \Lambda_{j}^{+}-\Lambda_{j}^{+} & 0 \\
0 & e^{-\mu \Delta x} \Lambda_{j}^{-}-\Lambda_{j}^{-}
\end{array}\right]\left[\begin{array}{c}
W_{j}^{+n} \\
W_{j}^{-n}
\end{array}\right] \\
& +\sum_{j=0}^{J-1}\left[\begin{array}{c}
W_{j}^{+n} \\
W_{j}^{-n}
\end{array}\right]^{T} \Phi_{j}\left[\begin{array}{cc}
-\Lambda_{j-1}^{+}+\Lambda_{j}^{+} & 0 \\
0 & -\Lambda_{j+1}^{-}+\Lambda_{j}^{-}
\end{array}\right]\left[\begin{array}{c}
W_{j}^{+n} \\
W_{j}^{-n}
\end{array}\right] \text {, } \\
& =-\mu e^{-\mu \Delta x} \Delta x \sum_{j=0}^{J-1}\left(W_{j}^{n}\right)^{T} \Phi_{j}\left|\Lambda_{j}\right|\left(W_{j}^{n}\right)+\Delta x \sum_{j=0}^{J-1}\left(W_{j}^{n}\right)^{T} \Phi_{j} \Lambda_{j}^{\prime}\left(W_{j}^{n}\right) .
\end{aligned}
$$

By assumption (16) and the condition: for $\alpha=\min _{\substack{1 \leq i \leq k \\ 0 \leq j \leq J-1}}\left|\lambda_{i, j}\right|$ and $\beta=\max _{\substack{1 \leq i \leq k \\ 0 \leq j \leq J-1}} \lambda_{i, j}^{\prime}$, such that $0<\mu \alpha e^{-\mu \Delta x}-\beta<1$, we have

$$
\frac{\mathcal{L}^{n+1}-\widetilde{\mathcal{L}}^{n}}{\Delta t} \leq 0
$$

and

$$
\frac{\widetilde{\mathcal{L}}^{n}-\mathcal{L}^{n}}{\Delta t}<-\eta \mathcal{L}^{n}
$$

with $\eta:=\mu \alpha e^{-\mu \Delta x}-\beta$. Thus,

$$
\frac{\mathcal{L}^{n+1}-\mathcal{L}^{n}}{\Delta t}<-\eta \mathcal{L}^{n}
$$

Recursively applying inequality (26) yields

$$
\mathcal{L}^{n+1}<(1-\Delta t \eta)^{n+1} \mathcal{L}^{0} \leq e^{-\eta \Delta t(n+1)} \mathcal{L}^{0}=e^{-\eta t^{n+1}} \mathcal{L}^{0}, \quad n=0, \ldots, N-1 .
$$

We use now the inequality (27) to conclude the proof. To this end, let

$$
C_{1}:=\min _{\substack{1 \leq i \leq k \\ 0 \leq j \leq J-1}}\left\{\sigma_{i, j}:\left|\Phi_{j}-\sigma_{i, j} I\right|=0\right\} \quad \text { and } \quad C_{2}:=\max _{\substack{1 \leq i \leq k \\ 0 \leq j \leq J-1}}\left\{\sigma_{i, j}:\left|\Phi_{j}-\sigma_{i, j} I\right|=0\right\} .
$$

Then,

$$
C_{1} I \leq \Phi_{j} \leq C_{2} I, \quad j=0, \ldots, J-1
$$


From inequalities (27) and (28), one obtains

$$
C_{1} \Delta x \sum_{j=0}^{J-1}\left[W_{j}^{n}\right]^{T}\left[W_{j}^{n}\right] \leq \mathcal{L}^{n} \leq C_{2} e^{-\eta t^{n}} \Delta x \sum_{j=0}^{J-1}\left[W_{j}^{0}\right]^{T}\left[W_{j}^{0}\right], \quad n=0, \ldots, N .
$$

It follows that

$$
\Delta x \sum_{j=0}^{J-1}\left[W_{j}^{n}\right]^{T}\left[W_{j}^{n}\right] \leq C e^{-\eta t^{n}} \Delta x \sum_{j=0}^{J-1}\left[W_{j}^{0}\right]^{T}\left[W_{j}^{0}\right], \quad n=0, \ldots, N
$$

where $C:=C_{2} / C_{1}$. Hence, the numerical solution $W_{j}^{n}$ of the Cauchy problem in (14) is exponentially stable for the $L^{2}-$ norm.

Stability conditions for non-uniform linear hyperbolic system with variable coefficients as presented in Theorem 3.2 above are analysed for specific examples in Section 4 below.

The special case of the system in Equation (10) for $n=0, \ldots, N-1$ is expressed as follows:

$$
\begin{aligned}
& {\left[\begin{array}{c}
\widetilde{W^{+}}{ }_{j}^{n} \\
\widetilde{W^{-}} \\
j
\end{array}\right]=\left[\begin{array}{c}
W_{j}^{+n} \\
W_{j}^{-n}
\end{array}\right]-\frac{\Delta t}{\Delta x}\left[\begin{array}{cc}
\Lambda^{+} & 0 \\
0 & \Lambda^{-}
\end{array}\right]\left[\begin{array}{c}
W_{j}^{+n}-W_{j-1}^{+n} \\
W_{j}^{-n}-W_{j+1}^{-n}
\end{array}\right], \quad n=0, \ldots, N-1,} \\
& {\left[\begin{array}{c}
W_{j}^{+n+1} \\
W_{j}^{-n+1}
\end{array}\right]=\left[\begin{array}{c}
\widetilde{W^{+}}{ }_{j}^{n} \\
\widetilde{W^{-}}{ }_{j}
\end{array}\right]-\Delta t \Pi\left[\begin{array}{c}
\widetilde{W^{+}}{ }^{n} \\
\widetilde{W^{-}} \\
j
\end{array}\right], \quad j=0, \ldots, J-1 .}
\end{aligned}
$$

A numerical stability analysis for discretised linear hyperbolic systems with constant coefficients (29) is also analysed as in Theorem 3.2 and given in the following corollary.

Corollary 3.3. Let $T>0$ and assume that $\lambda_{i}>0, i=1, \ldots, m$ and $\lambda_{i}<0$, $i=m+1, \ldots, k$. Let the Lyapunov function be given by (15). If the CFL condition $\frac{\Delta t}{\Delta x} \max _{1 \leq i \leq k}\left|\lambda_{i}\right| \leq 1$ holds and if there exists $\mu>0$ such that

$$
\Phi_{j} \Pi+\Pi^{T} \Phi_{j}-\Delta t \Pi^{T} \Phi_{j} \Pi \quad \text { is positive semi-definite }
$$

and

$$
\left[\begin{array}{cc}
P^{+} e^{-\mu x_{J}} \Lambda^{+} & 0 \\
0 & P^{-} e^{\mu x_{-1}} \Lambda^{-}
\end{array}\right]-K^{T}\left[\begin{array}{cc}
P^{+} e^{-\mu x_{0}} \Lambda^{+} & 0 \\
0 & P^{-} e^{\mu x_{J-1}} \Lambda^{-}
\end{array}\right] K,
$$

is positive definite for each $j=0, \ldots, J-1$, then the numerical solution $W_{j}^{n}$ of the Cauchy problem (14b), (14c), (29) satisfies

$$
\mathcal{L}^{n} \leq e^{-\eta t^{n}} \mathcal{L}^{0},
$$

for some $\eta>0$. Moreover, $W_{j}^{n}$ is exponentially stable for the $L^{2}-$ norm.

The proof of Corollary 3.3 uses the same idea applied in the proof of Theorem 3.2 with $\Lambda_{j}$ and $\Pi_{j}, j=0, \ldots, J-1$ considered as constant matrices. 
One of the conditions in Corollary 3.3 is to show that the matrix (31) is positive definite, which is alternatively described in the following proposition.

Proposition 3.4. Let $T>0$ and assume that $\lambda_{i}>0, i=1, \ldots, m$ and $\lambda_{i}<0$, $i=m+1, \ldots, k$. Let the discrete Lyapunov function be given by (15). If the CFL condition $\frac{\Delta t}{\Delta x} \max _{1 \leq i \leq k}\left|\lambda_{i}\right| \leq 1$ holds and if there exists $\mu>0$ such that the condition (30) holds and

$$
\left\|\Delta K \Delta^{-1}\right\|<1
$$

with $\Delta:=\sqrt{P|\Lambda|}$, where $P:=\operatorname{diag}\left\{P^{+}, P^{-}\right\}$, then the numerical solution $W_{j}^{n}$ of the Cauchy problem (29), (14b), (14c) satisfies the inequality (32) for some $\eta>0$. Moreover, $W_{j}^{n}$ is exponentially stable for the $L^{2}-$ norm.

Proof. The approximation of the time derivative of the Lyapunov function (7) under the CFL condition $\frac{\Delta t}{\Delta x} \max _{1 \leq i \leq k}\left|\lambda_{i}\right| \leq 1$ is given by (18) with (21) and

$$
\begin{aligned}
\frac{\widetilde{\mathcal{L}}^{n}-\mathcal{L}^{n}}{\Delta t} & <-\mu \alpha e^{-\mu \Delta x} \mathcal{L}^{n}+\left[\begin{array}{c}
W^{+n}{ }_{J-1} \\
W_{0}^{-n}
\end{array}\right]^{T} K^{T}\left[\begin{array}{cc}
P^{+} e^{-\mu x_{0}} \Lambda^{+} & 0 \\
0 & P^{-} e^{\mu x_{J-1}} \Lambda^{-}
\end{array}\right] K\left[\begin{array}{c}
W_{J-1}^{+n} \\
W^{-n} \\
0
\end{array}\right] \\
& -\left[\begin{array}{c}
W_{J-1}^{+n} \\
W^{-n} \\
0
\end{array}\right]^{T}\left[\begin{array}{cc}
P^{+} e^{-\mu x_{J}} \Lambda^{+} & 0 \\
0 & P^{-} e^{\mu x_{-1}} \Lambda^{-}
\end{array}\right]\left[\begin{array}{c}
W_{J-1}^{+n} \\
W_{0}^{-n} \\
0
\end{array}\right],
\end{aligned}
$$

where $\alpha:=\min _{1 \leq i \leq k}\left|\lambda_{i}\right|$.

By assumption (30), the inequality (24) holds. In order to show that the right hand 
side of the inequality (34) is negative, consider the following quadratic form

$$
\begin{aligned}
& -\left[\begin{array}{c}
W_{J-1}^{+n} \\
W_{0}^{-n}
\end{array}\right]^{T}\left[\begin{array}{cc}
\Lambda^{+} P^{+} & 0 \\
0 & \Lambda^{-} P^{-}
\end{array}\right]\left[\begin{array}{c}
W_{J-1}^{+n} \\
W_{0}^{-n}
\end{array}\right]+\left[\begin{array}{c}
W_{J-1}^{+n} \\
W^{-n}
\end{array}\right]^{T} K^{T}\left[\begin{array}{cc}
\Lambda^{+} P^{+} & 0 \\
0 & \Lambda^{-} P^{-}
\end{array}\right] K\left[\begin{array}{c}
W_{J-1}^{+n} \\
W^{-n} \\
0
\end{array}\right], \\
& =-\left[\begin{array}{c}
W_{J-1}^{+n} \\
W_{0}^{-n}
\end{array}\right]^{T}\left[\begin{array}{cc}
\Lambda^{+} P^{+} & 0 \\
0 & \Lambda^{-} P^{-}
\end{array}\right]\left[\begin{array}{c}
W_{J-1}^{+n} \\
W_{0}^{-n}
\end{array}\right] \\
& +\left[\begin{array}{c}
W_{J-1}^{+n} \\
W_{0}^{-n}
\end{array}\right]^{T} K^{T}(\sqrt{|\Lambda| P})^{T}(\sqrt{|\Lambda| P}) K\left[\begin{array}{c}
W_{J-1}^{+n} \\
W_{0}^{-n}
\end{array}\right] \text {, } \\
& =-\left[\begin{array}{c}
W_{J-1}^{+n} \\
W_{0}^{-n}
\end{array}\right]^{T}\left[\begin{array}{cc}
\Lambda^{+} P^{+} & 0 \\
0 & \Lambda^{-} P^{-}
\end{array}\right]\left[\begin{array}{c}
W_{J-1}^{+n} \\
W_{0}^{-n}
\end{array}\right]+\left(\sqrt{|\Lambda| P} K\left[\begin{array}{c}
W_{J-1}^{+n} \\
W_{0}^{-n}
\end{array}\right]\right)^{T}\left(\sqrt{|\Lambda| P} K\left[\begin{array}{c}
W_{J-1}^{+n} \\
W_{0}^{-n}
\end{array}\right]\right), \\
& =-\left[\begin{array}{c}
W_{J-1}^{+n} \\
W_{0}^{-n}
\end{array}\right]^{T}\left[\begin{array}{cc}
\Lambda^{+} P^{+} & 0 \\
0 & \Lambda^{-} P^{-}
\end{array}\right]\left[\begin{array}{c}
W_{J-1}^{+n} \\
W_{0}^{-n}
\end{array}\right]+\left\|\sqrt{|\Lambda| P} K\left[\begin{array}{c}
W_{J-1}^{+n} \\
W_{0}^{-n}
\end{array}\right]\right\|^{2} \text {, } \\
& =-\left[\begin{array}{c}
W_{J-1}^{+n} \\
W_{0}^{-n}
\end{array}\right]^{T}\left[\begin{array}{cc}
\Lambda^{+} P^{+} & 0 \\
0 & \Lambda^{-} P^{-}
\end{array}\right]\left[\begin{array}{c}
W_{J-1}^{+n} \\
W_{0}^{-n}
\end{array}\right] \\
& +\left\|(\sqrt{|\Lambda| P}) K(\sqrt{|\Lambda| P})^{-1}(\sqrt{|\Lambda| P})\left[\begin{array}{c}
W^{+n}{ }_{J-1} \\
W_{0}^{-n}
\end{array}\right]\right\|^{2}, \\
& \leq-\left[\begin{array}{c}
W_{J-1}^{+n} \\
W_{0}^{-n}
\end{array}\right]^{T}\left[\begin{array}{cc}
\Lambda^{+} P^{+} & 0 \\
0 & \Lambda^{-} P^{-}
\end{array}\right]\left[\begin{array}{c}
W_{J-1}^{+n} \\
W_{0}^{-n}
\end{array}\right] \\
& +\left\|(\sqrt{|\Lambda| P}) K(\sqrt{|\Lambda| P})^{-1}\right\|^{2}\left\|(\sqrt{|\Lambda| P})\left[\begin{array}{c}
W_{J-1}^{+n} \\
W_{0}^{-n}
\end{array}\right]\right\|^{2} \text {, }
\end{aligned}
$$

where in the last line, $p_{i}>0, i=1, \ldots, k$, can be selected such that

$$
\begin{aligned}
& \left\|(\sqrt{|\Lambda| P})\left[\begin{array}{c}
W^{+n} \\
J-1 \\
W_{0}^{-n}
\end{array}\right]\right\|=1 \quad \text { and } \quad\left[\begin{array}{c}
W_{J-1}^{+n} \\
W_{0}^{-n}
\end{array}\right] \neq 0, \quad n=0, \ldots, N-1 . \\
& \text { If }\left\|(\sqrt{|\Lambda| P}) K(\sqrt{|\Lambda| P})^{-1}\right\|<1 \text {, then } \\
& -\left[\begin{array}{c}
W_{J-1}^{+n} \\
W_{0}^{-n}
\end{array}\right]^{T}\left[\begin{array}{cc}
\Lambda^{+} P^{+} & 0 \\
0 & \Lambda^{-} P^{-}
\end{array}\right]\left[\begin{array}{c}
W_{J-1}^{+n} \\
W_{0}^{-n}
\end{array}\right]+\left[\begin{array}{c}
W_{J-1}^{+n} \\
W_{0}^{-n}
\end{array}\right]^{T} K^{T}\left[\begin{array}{cc}
\Lambda^{+} P^{+} & 0 \\
0 & \Lambda^{-} P^{-}
\end{array}\right] K\left[\begin{array}{c}
W_{J-1}^{+n} \\
W_{0}^{-n}
\end{array}\right], \\
& <-\left[\begin{array}{c}
W_{J-1}^{+n} \\
W_{0}^{-n}
\end{array}\right]^{T}|\Lambda| P\left[\begin{array}{c}
W_{J-1}^{+n} \\
W_{0}^{-n}
\end{array}\right]+\left\|\sqrt{|\Lambda| P}\left[\begin{array}{c}
W_{J-1}^{+n} \\
W_{0}^{-n}
\end{array}\right]\right\|^{2}, \\
& =-\left[\begin{array}{c}
W_{J-1}^{+n} \\
W_{0}^{-n}
\end{array}\right]^{T}|\Lambda| P\left[\begin{array}{c}
W_{J-1}^{+n} \\
W_{0}^{-n}
\end{array}\right]+\left[\begin{array}{c}
W_{J-1}^{+n} \\
W_{0}^{-n}
\end{array}\right]^{T}|\Lambda| P\left[\begin{array}{c}
W_{J-1}^{+n} \\
W_{0}^{-n}
\end{array}\right]=0 .
\end{aligned}
$$


Therefore, by assumption (33), a sufficiently small $\mu>0$ can be chosen such that the inequality (25) holds with $\eta:=\mu \alpha e^{-\mu \Delta x}$ and then the inequality (26) also holds. Hence, the conclusion of this proof follows from the proof of Theorem 3.2.

Remark 3. At this point it can be observed that through the analysis of the discrete problem a relationship between $\eta$ and $\mu$ has also been established.

\section{Applications}

In this section, we apply the numerical boundary stabilisation technique to some important examples of hyperbolic systems of balance laws in one-space dimension.

\subsection{Application to the Saint-Venant Equations}

Consider water flow along a prismatic channel with a rectangular cross-section, a length of $l$ units and constant bottom slope. In this case, the Saint-Venant Equations Bastin et al. (2008) is given by

$$
\begin{aligned}
& \partial_{t} H+\partial_{x}(H V)=0, \\
& \partial_{t} V+\partial_{x}\left(\frac{1}{2} V^{2}+g H\right)+\left(g C_{f} \frac{V^{2}}{H}-g S_{b}\right)=0, \quad x \in[0, l], \quad t \geq 0,
\end{aligned}
$$

where the physical quantities $H$ and $V$ represent the depth and velocity of the water, respectively, and the parameters in the source terms $g, C_{f}$ and $S_{b}$ represent gravitational constant, a friction parameter and the constant bottom slope of the channel, respectively, with initial conditions

$$
H(x, 0)=H_{0}(x), \quad V(x, 0)=V_{0}(x), \quad x \in[0, l],
$$

and boundary conditions

$$
V(0, t)=-k_{0} H(0, t), \quad V(l, t)=k_{l} H(l, t), \quad t \geq 0,
$$

where $k_{0}$ and $k_{l}$ are parameters.

The system (35) can be written in the form of non-uniform symmetric hyperbolic $2 \times 2$ system of balance laws (1) (The calculation is done in accordance with the discussion in Bastin \& Coron (2016)).

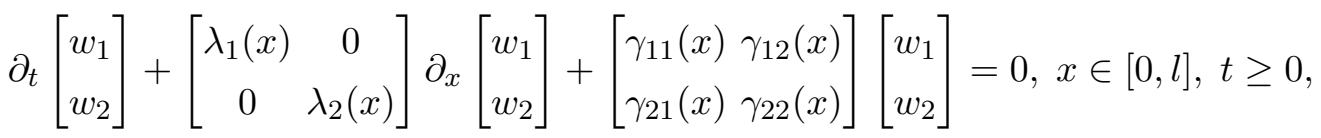


with

$$
\begin{aligned}
w_{1} & :=V-V^{*}(x)+\left(H-H^{*}(x)\right) \sqrt{\frac{g}{H^{*}(x)}}, \\
w_{2} & :=V-V^{*}(x)-\left(H-H^{*}(x)\right) \sqrt{\frac{g}{H^{*}(x)}}, \\
\lambda_{1}(x) & :=V^{*}(x)+\sqrt{g H^{*}(x)}, \quad \lambda_{2}(x):=V^{*}(x)-\sqrt{g H^{*}(x)}, \\
\gamma_{11}(x) & :=\frac{3}{4} \frac{g}{H^{*}(x)}\left(\frac{S_{b} H^{*}(x)-C_{f} V^{* 2}(x)}{\lambda_{1}(x)}\right)+\frac{g C_{f} V^{* 2}(x)}{2 H^{*}(x)}\left(\frac{2}{V^{*}(x)}-\frac{1}{\sqrt{g H^{*}(x)}}\right), \\
\gamma_{12}(x) & :=\frac{1}{4} \frac{g}{H^{*}(x)}\left(\frac{S_{b} H^{*}(x)-C_{f} V^{* 2}(x)}{\lambda_{1}(x)}\right)+\frac{g C_{f} V^{* 2}(x)}{2 H^{*}(x)}\left(\frac{2}{V^{*}(x)}+\frac{1}{\sqrt{g H^{*}(x)}}\right), \\
\gamma_{21}(x) & :=\frac{1}{4} \frac{g}{H^{*}(x)}\left(\frac{S_{b} H^{*}(x)-C_{f} V^{* 2}(x)}{\lambda_{2}(x)}\right)+\frac{g C_{f} V^{* 2}(x)}{2 H^{*}(x)}\left(\frac{2}{V^{*}(x)}-\frac{1}{\sqrt{g H^{*}(x)}}\right), \\
\gamma_{22}(x) & :=\frac{3}{4} \frac{g}{H^{*}(x)}\left(\frac{S_{b} H^{*}(x)-C_{f} V^{* 2}(x)}{\lambda_{2}(x)}\right)+\frac{g C_{f} V^{* 2}(x)}{2 H^{*}(x)}\left(\frac{2}{V^{*}(x)}+\frac{1}{\sqrt{g H^{*}(x)}}\right),
\end{aligned}
$$

where $H^{*}(x), V^{*}(x)$ is an equilibrium solution.

The initial condition (36) and boundary conditions (37) in the new coordinates can be expressed in the following form

$$
\left[\begin{array}{l}
w_{1}(x, 0) \\
w_{2}(x, 0)
\end{array}\right]:=\left[\begin{array}{l}
\left(V_{0}(x)-V^{*}(x)\right)+\left(H_{0}(x)-H^{*}(x)\right) \sqrt{\frac{g}{H^{*}(x)}} \\
\left(V_{0}(x)-V^{*}(x)\right)-\left(H_{0}(x)-H^{*}(x)\right) \sqrt{\frac{g}{H^{*}(x)}}
\end{array}\right], \quad x \in[0, l],
$$

and

$$
\left[\begin{array}{l}
w_{1}(0, t) \\
w_{2}(l, t)
\end{array}\right]=\left[\begin{array}{cc}
0 & k_{12} \\
k_{21} & 0
\end{array}\right]\left[\begin{array}{l}
w_{1}(l, t) \\
w_{2}(0, t)
\end{array}\right], t \geq 0
$$

with

$$
k_{12}:=\frac{k_{0}-\sqrt{\frac{g}{H^{*}(0)}}}{k_{0}+\sqrt{\frac{g}{H^{*}(0)}}}, \quad k_{21}:=\frac{k_{l}-\sqrt{\frac{g}{H^{*}(l)}}}{k_{l}+\sqrt{\frac{g}{H^{*}(l)}}} .
$$

The discretisation of the linear system (38), initial condition (39) and boundary conditions (40) is given by (14a), (14b) and

$$
\left[\begin{array}{l}
w_{1-1}^{n+1} \\
w_{2}^{n+1}
\end{array}\right]=\left[\begin{array}{cc}
0 & k_{12} \\
k_{21} & 0
\end{array}\right]\left[\begin{array}{l}
w_{1}^{n+1} \\
w_{20}^{n+1}
\end{array}\right], \quad n=0, \ldots, N-1,
$$

respectively.

The discrete Lyapunov function for the discretised Cauchy problem (14a), (14b), (41) is defined by (15) and the approximation of the time derivative of the Lyapunov 
function is given by (18) with (21). Thus, if the CFL condition

$$
\frac{\Delta t}{\Delta x} \max _{0 \leq j \leq J-1}\left\{\left|\lambda_{1, j}\right|,\left|\lambda_{2, j}\right|\right\} \leq 1
$$

holds and if there exists $\mu>0, p_{1}>0$ and $p_{2}>0$ such that the condition (16) holds and

$$
\begin{aligned}
& {\left[\begin{array}{cc}
p_{1} e^{-\mu x_{J}}\left|\lambda_{1, J-1}\right| & 0 \\
0 & p_{2} e^{\mu x_{-1}}\left|\lambda_{2,0}\right|
\end{array}\right]} \\
& -\left[\begin{array}{cc}
0 & k_{12} \\
k_{21} & 0
\end{array}\right]^{T}\left[\begin{array}{cc}
p_{1} e^{-\mu x_{0}}\left|\lambda_{1,-1}\right| & 0 \\
0 & p_{2} e^{\mu x_{J-1}}\left|\lambda_{2, J}\right|
\end{array}\right]\left[\begin{array}{cc}
0 & k_{12} \\
k_{21} & 0
\end{array}\right]
\end{aligned}
$$

is positive definite, then both inequalities (24) and (25) hold with

$$
\begin{gathered}
0<\eta:=\mu \alpha e^{-\mu \Delta x}-\beta<1, \quad \alpha:=\min _{0 \leq j \leq J-1}\left\{\left|\lambda_{1, j}\right|,\left|\lambda_{2, j}\right|\right\}, \\
\beta:=\max _{0 \leq j \leq J-1}\left\{\frac{\lambda_{1, j}-\lambda_{1, j-1}}{\Delta x}, \frac{\lambda_{2, j+1}-\lambda_{2, j}}{\Delta x}\right\} .
\end{gathered}
$$

In order to show that both conditions (16) and (42) hold, it suffices to show that the determinant of every principal sub-matrix of the matrices

$$
\left[\begin{array}{ll}
M_{11, j} & M_{12, j} \\
M_{12, j} & M_{22, j}
\end{array}\right],
$$

with

$$
\begin{aligned}
& M_{11, j}:=2 p_{1} e^{-\mu x_{j}} \gamma_{11, j}-\Delta t\left(p_{1} e^{-\mu x_{j}} \gamma_{11, j}^{2}+p_{2} e^{\mu x_{j}} \gamma_{21, j}^{2}\right), \\
& M_{12, j}:=p_{2} e^{\mu x_{j}} \gamma_{21, j}+p_{1} e^{-\mu x_{j}} \gamma_{12, j}-\Delta t\left(p_{1} e^{-\mu x_{j}} \gamma_{11, j} \gamma_{12, j}+p_{2} e^{\mu x_{j}} \gamma_{21, j} \gamma_{22, j}\right), \\
& M_{22, j}:=2 p_{2} e^{\mu x_{j}} \gamma_{22, j}-\Delta t\left(p_{1} e^{-\mu x_{j}} \gamma_{12, j}^{2}+p_{2} e^{\mu x_{j}} \gamma_{22, j}^{2}\right),
\end{aligned}
$$

and

$$
\operatorname{diag}\left\{p_{1} e^{-\mu x_{J}}\left|\lambda_{1, J-1}\right|-k_{21}^{2} p_{2} e^{\mu x_{J-1}}\left|\lambda_{2, J}\right|, p_{2} e^{\mu x_{-1}}\left|\lambda_{2,0}\right|-k_{12}^{2} p_{1} e^{-\mu x_{0}}\left|\lambda_{1,-1}\right|\right\},
$$

is non-negative for $0 \leq j \leq J-1$ and positive, respectively.

The determinant of the sub-matrices of the matrix (43) are

$$
M_{11, j}=2 p_{1} e^{-\mu x_{j}} \gamma_{11, j}-\Delta t\left(p_{1} e^{-\mu x_{j}} \gamma_{11, j}^{2}+p_{2} e^{\mu x_{j}} \gamma_{21, j}^{2}\right), \quad 0 \leq j \leq J-1,
$$


and

$$
\begin{aligned}
M_{11, j} M_{22, j}- & M_{11, j}^{2}=\Delta t^{2} \gamma_{11, j}^{2} \gamma_{22, j}^{2} p_{1} p_{2}-2 \Delta t^{2} \gamma_{11, j} \gamma_{12, j} \gamma_{21, j} \gamma_{22, j} p_{1} p_{2} \\
& +\Delta t^{2} \gamma_{12, j}^{2} \gamma_{21, j}^{2} p_{1} p_{2} \\
& -2 \Delta t \gamma_{11, j}^{2} \gamma_{22, j} p_{1} p_{2}+2 \Delta t \gamma_{11, j} \gamma_{12, j} \gamma_{21, j} p_{1} p_{2}-2 \Delta t \gamma_{11, j} \gamma_{22, j}^{2} p_{1} p_{2} \\
& +2 \Delta t \gamma_{12, j} \gamma_{21, j} \gamma_{22, j} p_{1} p_{2}+4 \gamma_{11, j} \gamma_{22, j} p_{1} p_{2} \\
& -e^{-2 \mu x_{j}} \gamma_{12, j}^{2} p_{1}^{2}-2 \gamma_{12, j} \gamma_{21, j} p_{1} p_{2}-e^{2 \mu x_{j}} \gamma_{21, j}^{2} p_{2}^{2} \\
& =\Delta t^{2}\left(\gamma_{11, j} \gamma_{22, j}-\gamma_{12, j} \gamma_{21, j}\right)^{2} p_{1} p_{2} \\
& -2 \Delta t\left(\gamma_{11, j}+\gamma_{22, j}\right)\left(\gamma_{11, j} \gamma_{22, j}-\gamma_{12, j} \gamma_{21, j}\right) p_{1} p_{2} \\
& +4\left(\gamma_{11, j} \gamma_{22, j}-\gamma_{12, j} \gamma_{21, j}\right) p_{1} p_{2}-\left(p_{1} e^{-\mu x_{j}} \gamma_{12, j}-p_{2} e^{\mu x_{j}} \gamma_{21, j}\right)^{2} \\
& =\Delta t^{2}\left(\gamma_{11, j} \gamma_{22, j}-\gamma_{12, j} \gamma_{21, j}\right)^{2} p_{1} p_{2}-\left(p_{1} e^{-\mu x_{j}} \gamma_{12, j}-p_{2} e^{\mu x_{j}} \gamma_{21, j}\right)^{2} \\
& +\left(4-2 \Delta t\left(\gamma_{11, j}+\gamma_{22, j}\right)\right)\left(\gamma_{11, j} \gamma_{22, j}-\gamma_{12, j} \gamma_{21, j}\right) p_{1} p_{2}, \quad 0 \leq j \leq J-1 .
\end{aligned}
$$

For $i=0, \ldots, J-1$, and sufficiently small $\mu>0$, if

$$
\gamma_{11, j} \gamma_{22, j}-\gamma_{12, j} \gamma_{21, j}>0, \quad \gamma_{11, j}>0, \quad \gamma_{12, j}>0, \quad \gamma_{21, j}>0, \quad \gamma_{22, j}>0
$$

and $\delta t<\frac{2}{\gamma_{11, j}+\gamma_{22, j}}$, and if $p_{1}$ and $p_{2}$ can be chosen such that

$$
\begin{gathered}
\frac{p_{1}}{p_{2}}=\max _{0 \leq j \leq J-1}\left\{\frac{\delta t \gamma_{21, j}^{2} e^{2 \mu x_{j}}}{\left(2-\delta t \gamma_{11, j}\right) \gamma_{11, j}}\right\}, \quad p_{1}=\left[\max _{0 \leq j \leq J-1} \gamma_{12, j}\right]^{-1}, \\
\text { and } p_{2}=\left[\max _{0 \leq j \leq J-1} \gamma_{21, j}\right]^{-1},
\end{gathered}
$$

then the determinants (45) and (46) are non-negative.

Therefore, with the choice of $p_{1}$ and $p_{2}$, the matrix (44) is positive definite if $k_{12}$ and $k_{21}$ satisfy

$$
\left|k_{12}\right|<\sqrt{\left|\frac{\lambda_{2,0}}{\lambda_{1,-1}}\right| \frac{p_{2}}{p_{1}}} \text { and }\left|k_{21}\right|<\sqrt{\left|\frac{\lambda_{1, J-1}}{\lambda_{2, J}}\right| \frac{p_{1}}{p_{2}}} e^{-\mu l}
$$

respectively.

To simplify the numerical computations, we take a constant steady state,

$$
H^{*}(x)=2, \quad V^{*}(x)=3,
$$

for $x \in[0, l]$, from A. Diagne, Diagne, Tang, \& Krstic (2017); M. Diagne, Tang, Diagne, $\&$ Krstic (2017) with physical parameters $g=9.81, C_{f}=0.1$ and $S_{b}=0.0459$ and initial condition for the system (35),

$$
H(x, 0)=2.5, \quad V(x, 0)=4 \sin (\pi x), \text { for } x \in[0,1] .
$$

The linear system has eigenvalues, $\lambda_{1}=7.4294$ and $\lambda_{2}=-1.4294$ and coefficients of the source terms are $\gamma_{11}=\gamma_{21}=0.0992$ and $\gamma_{12}=\gamma_{22}=0.2008$. 
We set an initial condition,

$$
w_{1}(x, 0)=-1.8926+4 \sin (\pi x), \quad w_{2}(x, 0)=-4.1074+4 \sin (\pi x),
$$

for $x \in[0,1]$.

The condition for the decay rate is $0<\eta:=\alpha \mu e^{-\mu \Delta x}-\beta<1$ where $\alpha=1.4294$ and $\beta=0$ and the spatial gridsize is $\Delta x=1 / 200=0.05$ with $\mathrm{CFL}=0.75$. This implies $0<\mu<0.7021$ or $\mu>1539.26$. For numerical implementation, a sufficiently small value of $\mu$ is chosen such that for the constant steady state the parameters $p_{1}$ and $p_{2}$ are chosen such that $p_{1} \gamma_{12}=p_{2} \gamma_{21}$. For this example, the values $p_{1}=\gamma_{21}=0.0992$ and $p_{2}=\gamma_{12}=0.2008$ were used. With this choice of parameters, we obtain $\left|k_{12}\right|<0.6241$ and $\left|k_{21}\right|<1.6024 e^{-\mu}<1.6024$. The numerical convergence of the discrete Lyapunov function for different values of $\mu>0$ is shown in Figure 1 below.

The three curves that are obtained for different values of $\mu>0$, which are shown in Figure 1, are nearly indistinguishable asymptotically in time. This shows that in the sense of the $L^{2}$-norm, the Cauchy problem (14a), (14b), (41) is exponentially stable for a preferred equilibrium solution, $W^{*}(x) \equiv 0\left(W_{j}^{n} \equiv 0\right)$.

Similar tests were also applied to the isentropic Euler equations (see also Weldegiyorgis (2017)). As expected, the behaviour of the Lyapunov function is similar.

\subsection{Application to the Saint-Venant-Exner Equations}

Consider the transport of sediments in a water flow along the prismatic channel with rectangular cross-section and constant bottom slope where the sediment moves predominantly as bed load. In this case, the Saint-Venant-Exner equations A. Diagne et al. (2012) results from coupling the Exner equation and the Saint-Venant equations, which is given by

$$
\begin{aligned}
& \partial_{t} H+\partial_{x}(H V)=0, \\
& \partial_{t} V+\partial_{x}\left(\frac{1}{2} V^{2}+g(H+B)\right)+\left(C_{f} \frac{V^{2}}{H}-g S_{b}\right)=0, \quad x \in[0, l], \quad t \geq 0, \\
& \partial_{t} B+\partial_{x}\left(\frac{1}{3} a V^{3}\right)=0,
\end{aligned}
$$

where the physical quantities $H, V$ and $B$ represent the depth of the water, the velocity of the water, and the bathymetry, respectively. The parameters in source term, $g, S_{b}$ and $C_{f}$ represent the gravitational constant, the bottom slope of the channel and a friction coefficient, respectively, with a parameter $a$, that encompasses porosity and viscosity effects on the sediment dynamics. We set the following initial condition

$$
[H(x, 0), V(x, 0), B(x, 0)]^{T}=\left[H_{0}(x), V_{0}(x), B_{0}(x)\right]^{T}, \quad x \in[0, l],
$$

and boundary conditions

$$
V(0, t)=-k_{0} H(0, t), \quad V(l, t)=-k_{l}(H(l, t)+B(l, t)) \quad \text { and } \quad B(0, t)=0, \quad t \geq 0,
$$

where $k_{0}$ and $k_{l}$ are parameters, for the system (47).

The system (47) can be written in the form of symmetric hyperbolic $3 \times 3$ system 
of balance laws (10). See also, A. Diagne et al. (2012).

$$
\partial_{t}\left[\begin{array}{l}
w_{1} \\
w_{2} \\
w_{3}
\end{array}\right]+\left[\begin{array}{ccc}
\lambda_{1} & 0 & 0 \\
0 & \lambda_{2} & 0 \\
0 & 0 & \lambda_{3}
\end{array}\right] \partial_{x}\left[\begin{array}{l}
w_{1} \\
w_{2} \\
w_{3}
\end{array}\right]+\left[\begin{array}{ccc}
\gamma_{1} & \gamma_{2} & \gamma_{3} \\
\gamma_{1} & \gamma_{2} & \gamma_{3} \\
\gamma_{1} & \gamma_{2} & \gamma_{3}
\end{array}\right]\left[\begin{array}{c}
w_{1} \\
w_{2} \\
w_{3}
\end{array}\right]=0, x \in[0, l], t \geq 0
$$

where the eigenvalues, $\lambda_{1}, \lambda_{2}, \lambda_{3}$ can be obtained by using Cardano-Vieta method Hudson \& Sweby (2003), which are roots of a cubic equation, $\lambda^{3}-2 V^{*} \lambda^{2}+\left(V^{* 2}-\right.$ $\left.g\left(H^{*}+V^{* 2} a\right)\right) \lambda+V^{* 3} a g=0$, with corresponding left eigenvectors as given in A. Diagne et al. (2012),

$$
L_{i}=\frac{1}{\left(\lambda_{i}-\lambda_{j}\right)\left(\lambda_{i}-\lambda_{k}\right)}\left[\left(V-\lambda_{j}\right)\left(V-\lambda_{k}\right)+g H^{*}, H \lambda_{i}, g H\right]
$$

for $i \neq j \neq k \in\{1,2,3\}$, and the new coordinates are defined by, $w_{i}:=\frac{1}{\delta_{i}} \xi_{i}$, $\xi_{i}:=L_{i}\left[H-H^{*}, V-V^{*}, B-B^{*}\right]^{T}$,

$$
\delta_{i}:=\left(C_{f} \frac{V^{*}}{H^{*}}\right)\left(\frac{\lambda_{i}}{\left(\lambda_{i}-\lambda_{j}\right)\left(\lambda_{i}-\lambda_{k}\right)}\right),
$$

for $i \neq j \neq k \in\{1,2,3\}$. Furthermore, the coefficients of the source terms are,

$$
\begin{gathered}
\gamma_{1}=\frac{\lambda_{1}\left(2 \lambda_{1}-3 V^{*}\right)}{\left(\lambda_{1}-\lambda_{2}\right)\left(\lambda_{1}-\lambda_{3}\right)}\left(C_{f} \frac{V^{*}}{H^{*}}\right), \quad \gamma_{2}=\frac{\lambda_{2}\left(2 \lambda_{2}-3 V^{*}\right)}{\left(\lambda_{2}-\lambda_{1}\right)\left(\lambda_{2}-\lambda_{3}\right)}\left(C_{f} \frac{V^{*}}{H^{*}}\right), \\
\gamma_{3}=\frac{\lambda_{3}\left(2 \lambda_{3}-3 V^{*}\right)}{\left(\lambda_{3}-\lambda_{1}\right)\left(\lambda_{3}-\lambda_{2}\right)}\left(C_{f} \frac{V^{*}}{H^{*}}\right),
\end{gathered}
$$

where $H^{*}, V^{*}, B^{*}$ is an equilibrium solution.

The initial condition (48) and boundary conditions (49) in the new coordinates can be expressed in the following form

$$
\left[\begin{array}{c}
w_{1}(x, 0) \\
w_{2}(x, 0) \\
w_{3}(x, 0)
\end{array}\right]=\left[\begin{array}{c}
\frac{1}{\delta_{1}} L_{1} \\
\frac{1}{\delta_{2}} L_{2} \\
\frac{1}{\delta_{3}} L_{3}
\end{array}\right]\left[\begin{array}{c}
H(x, 0)-H^{*} \\
V(x, 0)-V^{*} \\
B(x, 0)-B^{*}
\end{array}\right], \quad x \in[0, l]
$$

and

$$
\left[\begin{array}{l}
w_{1}(0, t) \\
w_{2}(0, t) \\
w_{3}(l, t)
\end{array}\right]=\left[\begin{array}{ccc}
0 & 0 & k_{13} \\
0 & 0 & k_{23} \\
k_{31} & k_{32} & 0
\end{array}\right]\left[\begin{array}{l}
w_{1}(l, t) \\
w_{2}(l, t) \\
w_{3}(0, t)
\end{array}\right], \quad t \geq 0
$$


with

$$
\begin{aligned}
k_{13}:=\frac{\delta_{3} L_{1} \zeta_{1}}{\delta_{1} L_{3} \zeta_{1}}, \quad k_{23} & :=\frac{\delta_{3} L_{2} \zeta_{1}}{\delta_{2} L_{3} \zeta_{1}}, \quad k_{31}:=\frac{\delta_{1}\left(L_{2} \zeta_{2} L_{3} \zeta_{3}-L_{3} \zeta_{2} L_{2} \zeta_{3}\right)}{\delta_{3}\left(L_{2} \zeta_{2} L_{1} \zeta_{3}-L_{1} \zeta_{2} L_{2} \zeta_{3}\right)} \\
k_{32} & :=\frac{-\delta_{2}\left(L_{1} \zeta_{2} L_{3} \zeta_{3}-L_{3} \zeta_{2} L_{1} \zeta_{3}\right)}{\delta_{3}\left(L_{2} \zeta_{2} L_{1} \zeta_{3}-L_{1} \zeta_{2} L_{2} \zeta_{3}\right)}
\end{aligned}
$$

where $\zeta_{1}:=\left[1,-k_{0}, 0\right]^{T}, \zeta_{2}:=\left[0,-k_{l}, 1\right]^{T}$ and $\zeta_{3}:=\left[1,-k_{l}, 0\right]^{T}$.

The discretisation of the linear system (50) and initial condition (51) is given by (29) and (14b), respectively, with discretised boundary conditions (52) expressed as follows

$$
\left[\begin{array}{l}
w_{1-1}^{n+1} \\
w_{2-1}^{n+1} \\
w_{3}^{n+1}
\end{array}\right]=\left[\begin{array}{ccc}
0 & 0 & k_{13} \\
0 & 0 & k_{23} \\
k_{31} & k_{32} & 0
\end{array}\right]\left[\begin{array}{l}
w_{1}^{n+1} \\
w_{2-1}^{n+1} \\
w_{30}^{n+1}
\end{array}\right], \quad n=0, \ldots, N-1 .
$$

The discrete Lyapunov function for the discretised Cauchy problem (29), (14b), (53) is defined by (15) and the approximation of the time derivative of the Lyapunov function is given by (18) with (21). Thus, if the CFL condition $\frac{\Delta t}{\Delta x} \max \left\{\left|\lambda_{1}\right|,\left|\lambda_{2}\right|,\left|\lambda_{3}\right|\right\} \leq 1$ holds and if there exists $\mu>0, p_{1}>0, p_{2}>0$ and $p_{3}>0$ such that the condition (30) holds and

$$
\begin{aligned}
& {\left[\begin{array}{ccc}
p_{1} e^{-\mu x_{J}}\left|\lambda_{1}\right| & 0 & 0 \\
0 & p_{2} e^{-\mu x_{J}}\left|\lambda_{2}\right| & 0 \\
0 & 0 & p_{3} e^{\mu x_{-1}}\left|\lambda_{3}\right|
\end{array}\right]} \\
& -\left[\begin{array}{ccc}
0 & 0 & k_{13} \\
0 & 0 & k_{23} \\
k_{31} & k_{32} & 0
\end{array}\right]^{T}\left[\begin{array}{ccc}
p_{1} e^{-\mu x_{0}}\left|\lambda_{1}\right| & 0 & 0 \\
0 & p_{2} e^{-\mu x_{0}}\left|\lambda_{2}\right| & 0 \\
0 & 0 & p_{3} e^{\mu x_{J-1}}\left|\lambda_{3}\right|
\end{array}\right]\left[\begin{array}{ccc}
0 & 0 & k_{13} \\
0 & 0 & k_{23} \\
k_{31} & k_{32} & 0
\end{array}\right]
\end{aligned}
$$

is positive definite, then both inequalities (24) and (25) hold with

$$
0<\eta:=\mu \alpha e^{-\mu \Delta x}<1, \quad \alpha:=\min \left\{\left|\lambda_{1}\right|,\left|\lambda_{2}\right|,\left|\lambda_{3}\right|\right\} .
$$

In order to show that both conditions (30) and (54) hold, it suffices to show that the determinant of every principal sub-matrix of the matrices

$$
\left[\begin{array}{lll}
M_{11} & M_{12} & M_{13} \\
M_{12} & M_{22} & M_{23} \\
M_{13} & M_{23} & M_{33}
\end{array}\right]
$$


with

$$
\begin{aligned}
& M_{11}:=2 p_{1} e^{-\mu x_{j}} \gamma_{1}-\Delta t\left(p_{1} e^{-\mu x_{j}} \gamma_{1}^{2}+p_{2} e^{-\mu x_{j}} \gamma_{1}^{2}+p_{3} e^{\mu x_{j}} \gamma_{1}^{2}\right), \\
& M_{12}:=p_{2} e^{-\mu x_{j}} \gamma_{1}+p_{1} e^{-\mu x_{j}} \gamma_{2}-\Delta t\left(p_{1} e^{-\mu x_{j}} \gamma_{1} \gamma_{2}+p_{2} e^{-\mu x_{j}} \gamma_{1} \gamma_{2}+p_{3} e^{\mu x_{j}} \gamma_{1} \gamma_{2}\right), \\
& M_{13}:=p_{3} e^{\mu x_{j}} \gamma_{1}+p_{1} e^{-\mu x_{j}} \gamma_{3}-\Delta t\left(p_{1} e^{-\mu x_{j}} \gamma_{1} \gamma_{3}+p_{2} e^{-\mu x_{j}} \gamma_{1} \gamma_{3}+p_{3} e^{\mu x_{j}} \gamma_{1} \gamma_{3}\right), \\
& M_{22}:=2 p_{2} e^{-\mu x_{j}} \gamma_{2}-\Delta t\left(p_{1} e^{-\mu x_{j}} \gamma_{2}^{2}+p_{2} e^{-\mu x_{j}} \gamma_{2}^{2}+p_{3} e^{\mu x_{j}} \gamma_{2}^{2}\right), \\
& M_{23}:=p_{3} e^{\mu x_{j}} \gamma_{2}+p_{2} e^{-\mu x_{j}} \gamma_{3}-\Delta t\left(p_{1} e^{-\mu x_{j}} \gamma_{2} \gamma_{3}+p_{2} e^{-\mu x_{j}} \gamma_{2} \gamma_{3}+p_{3} e^{\mu x_{j}} \gamma_{2} \gamma_{3}\right), \\
& M_{33}:=2 p_{3} e^{\mu x_{j}} \gamma_{3}-\Delta t\left(p_{1} e^{-\mu x_{j}} \gamma_{3}^{2}+p_{2} e^{-\mu x_{j}} \gamma_{3}^{2}+p_{3} e^{\mu x_{j}} \gamma_{3}^{2}\right),
\end{aligned}
$$

and

$$
\left[\begin{array}{ccc}
\sigma_{11} & \sigma_{12} & 0 \\
\sigma_{12} & \sigma_{22} & 0 \\
0 & 0 & \sigma_{33}
\end{array}\right]
$$

with

$$
\begin{gathered}
\sigma_{11}:=\left|\lambda_{1}\right| p_{1} e^{-\mu x_{J}}-k_{31}^{2}\left|\lambda_{3}\right| p_{3} e^{\mu x_{J-1}}, \quad \sigma_{12}:=-k_{31} k_{32}\left|\lambda_{3}\right| p_{3} e^{\mu x_{J-1}}, \\
\sigma_{22}:=\left|\lambda_{2}\right| p_{2} e^{-\mu x_{J}}-k_{32}^{2}\left|\lambda_{3}\right| p_{3} e^{\mu x_{J-1}}, \\
\sigma_{33}:=\left|\lambda_{3}\right| p_{3} e^{\mu x_{-1}}-k_{13}^{2}\left|\lambda_{1}\right| p_{1} e^{-\mu x_{0}}-k_{23}^{2}\left|\lambda_{2}\right| p_{2} e^{-\mu x_{0}},
\end{gathered}
$$

is non-negative and positive, respectively.

The determinant of the sub-matrices of the matrix (55) are

$$
\begin{gathered}
2 p_{1} e^{-\mu x_{j}} \gamma_{1}-\Delta t\left(p_{1} e^{-\mu x_{j}} \gamma_{1}^{2}+p_{2} e^{-\mu x_{j}} \gamma_{1}^{2}+p_{3} e^{\mu x_{j}} \gamma_{1}^{2}\right), \\
-\left(\gamma_{1}^{2} p_{2}^{2}-2 \gamma_{1} \gamma_{2} p_{1} p_{2}+\gamma_{2}^{2} p_{1}^{2}\right) e^{-2 \mu x_{j}}=-\left(\gamma_{1} p_{2}-\gamma_{2} p_{1}\right)^{2} e^{-2 \mu x_{j}},
\end{gathered}
$$

and

$$
\left|\begin{array}{lll}
M_{11} & M_{12} & M_{13} \\
M_{12} & M_{22} & M_{23} \\
M_{13} & M_{23} & M_{33}
\end{array}\right|=0 .
$$

If $p_{1}, p_{2}$ and $p_{3}$ can be chosen such that $p_{1}=\gamma_{1}, p_{2}=\gamma_{2}$ and $p_{3}=\gamma_{3}$, then for $\gamma_{1}>0$ and sufficiently small $\mu>0$,

$$
\Delta t \gamma_{1}<\max _{0 \leq x \leq l}\left\{\frac{2 p_{1} e^{-\mu x_{j}}}{\left(p_{1} e^{-\mu x_{j}}+p_{2} e^{-\mu x_{j}}+p_{3} e^{\mu x_{j}}\right)}\right\} \approx 1,
$$

and the determinants (57), (58) and (59) are non-negative. Therefore, with the choice of $p_{1}$ and $p_{2}$, the matrix (56) is positive definite if $k_{13}, k_{23}, k_{31}$ and $k_{32}$ satisfy,

$$
\left|k_{13}\right|<\sqrt{\left|\frac{\lambda_{3}}{\lambda_{1}}\right| \frac{\gamma_{3}}{\gamma_{1}}}, \quad\left|k_{23}\right|<\sqrt{\left|\frac{\lambda_{3}}{\lambda_{2}}\right| \frac{\gamma_{3}}{\gamma_{2}}}, \quad\left|k_{31}\right|<\sqrt{\left|\frac{\lambda_{1}}{\lambda_{3}}\right| \frac{\gamma_{1}}{\gamma_{3}}} e^{-\mu l}, \quad\left|k_{32}\right|<\sqrt{\left|\frac{\lambda_{2}}{\lambda_{3}}\right| \frac{\gamma_{2}}{\gamma_{3}}} e^{-\mu l} .
$$


Consider a constant steady state A. Diagne et al. (2017); M. Diagne et al. (2017),

$$
H^{*}(x)=2, \quad V^{*}(x)=3, \quad B^{*}(x)=0.4,
$$

for $x \in[0,1]$, with physical parameter values $g=9.81, C_{f}=0.1, a=0.0184$ and $S_{b}=0.0459$ in the system (47). The initial condition for the system (47) is given by A. Diagne et al. (2017); M. Diagne et al. (2017)

$$
\begin{gathered}
H(x, 0)=2.5-B(x, 0), \quad V(x, 0)=\frac{10 \sin (\pi x)}{H(x, 0)}, \\
B(x, 0)=0.4\left(1+0.25 \exp \left(-\frac{(x-0.5)^{2}}{0.003}\right)\right)
\end{gathered}
$$

for $x \in[0,1]$. The linear system has eigenvalues $\lambda_{1}=7.5383, \lambda_{2}=0.3430$ and $\lambda_{3}=$ -1.8813 and the coefficients of the source terms are $\gamma_{1}=0.1014, \gamma_{2}=0.0267$ and $\gamma_{3}=0.1719$. The initial condition, which is obtained from (51), is given by:

$$
w_{1}(x, 0)=-1.8926+4 \sin (\pi x), \quad w_{2}(x, 0)=-4.1074+4 \sin (\pi x),
$$

for $x \in[0,1]$.

The condition for the numerical decay rate is $0<\eta:=\alpha \mu e^{-\mu}<1$ where $\alpha=1.8813$, the spatial gridsize is $\Delta x=1 / 200=0.005$ with $\mathrm{CFL}=0.75$. This gives $0<\mu<$ 0.532966 or $\mu>1602.22$. For numerical implementation we chose $p_{1}=\gamma_{1}=0.1014$, $p_{2}=\gamma_{2}=0.0267$ and $p_{3}=\gamma_{3}=0.1719$. With this choice of parameters, the control parameters can be chosen as $k_{13}<0.6504, k_{23}<5.9424, k_{31}<1.5374 e^{-\mu}<1.5374$ and $k_{32}<0.1683 e^{-\mu}<0.1683$. The convergence of the discrete Lyapunov function for different values of $\mu>0$ is shown in Figure 2 below.

The three curves that are obtained for different values of $\mu>0$, which are shown in the Figure 2, are nearly indistinguishable. In Figure 2, we observe the decay of the discrete Lyapunov function. This shows, in the sense of the $L^{2}$-norm, the Cauchy problem (29), (14b), (53) is exponentially stable for a preferred equilibrium solution, $W^{*}(x) \equiv 0\left(W_{j}^{n} \equiv 0\right)$.

\section{Conclusion}

In this paper, a linear hyperbolic system of balance laws has been considered and a finite volume method is used in the discretisation of this linear system. In particular, the upwind scheme and splitting method are used to discretise the space and time, respectively. Beside this, an $L^{2}$-Lyapunov function is discretised and used to investigate conditions for exponential stability of the discretised system. Furthermore, the result was applied to some relevant physical problems such as the telegrapher equations, isentropic Euler equations, Saint-Venant equations and Saint-Venant-Exner equations. In this article only the results from Saint-Venant and Saint-Venant-Exner equations were discussed. Finally, numerical simulations are computed in order to test the results and compare with analytical results.

As part of future work plans, the numerical stability analysis of the nonlinear hyperbolic systems of balance laws will be explored by using the $H^{2}$-Lyapunov function. 
Further to that an extension of the above analysis to a network has also been planned as future work.

\section{Acknowledgments}

G. Weldegiyorgis thanks Department of Mathematics and Applied Mathematics of University of Pretoria for partial funding for his research. M.K. Banda is also grateful to African Institute for Mathematical Sciences - South Africa and for hosting him while finalising this work. He is also grateful for DAAD funding (ID: 57314019) for a visit at RWTH-Aachen. This work is also supported in part by the National Research Foundation of South Africa (Grant number: 93099 and 93476).

\section{References}

Banda, M. K., \& Herty, M. (2013). Numerical discretization of stabilization problems with boundary controls for systems of hyperbolic conservation laws. Math. Control Relat. Fields, 3(2), 121-142.

Banda, M. K., Herty, M., \& Klar, A. (2006). Gas flow in pipeline networks. NHM, 1(1), 41-56.

Bastin, G., \& Coron, J. (2016). Stability and boundary stabilization of 1-d hyperbolic systems. Basel: Birkhäuser.

Bastin, G., \& Coron, J.-M. (2011). On boundary feedback stabilization of non-uniform linear $2 \times 2$ hyperbolic systems over a bounded interval. Systems \& Control Letters, 60(11), 900906.

Bastin, G., Coron, J.-M., \& d'Andréa Novel, B. (2008). Using hyperbolic systems of balance laws for modeling, control and stability analysis of physical networks. In Lecture notes for the pre-congress workshop on complex embedded and networked control systems, Seoul, Korea.

Bastin, G., Coron, J.-M., \& d'Andréa Novel, B. $\quad$ (2009). On Lyapunov stability of linearised Saint-Venant equations for a sloping channel. Networks and Heterogeneous Media, 4(2), 177-187. Retrieved from http: //aimsciences.org/journals/displayArticlesnew.jsp?paperID=4234 doi:

Chalons, C., Girardin, M., \& Kokh, S. (2012). Operator-splitting based AP schemes for the 1D and 2D gas dynamics equations with stiff sources. In Aims series on applied mathematics, vol 8, 607-614(2014); Proceedings of the 2012 International Conference on Hyperbolic Problems.

Coron, J.-M. (2007). Control and nonlinearity. Providence, Rhode Island: American Mathematical Society. Retrieved from https://books.google.co.za/books?id=aEKv1bpcrKQC

Coron, J.-M., Bastin, G., \& d'Andréa Novel, B. (2008). Dissipative boundary conditions for one-dimensional nonlinear hyperbolic systems. SIAM Journal on Control and Optimization, $47(3), 1460-1498$.

Coron, J.-M., d'Andréa Novel, B., \& Bastin, G. (2007). A strict Lyapunov function for boundary control of hyperbolic systems of conservation laws. Automatic Control, IEEE Transactions on, 52(1), 2-11.

Dafermos, C. (2009). Hyperbolic conservation laws in continuum physics. Springer Berlin Heidelberg. Retrieved from https://books.google.co.za/books?id=49bXK260_b4C

de Halleux, J., \& Bastin, G. (2002). Stabilization of St. Venant equations using Riemann invariants: Application to waterways with mobile spillways. IFAC Proceedings Volumes, 35(1), 131 - 136 . Retrieved from http://www.sciencedirect.com/science/article/pii/S1474667015399961 (15th IFAC World Congress) doi: 
de Halleux, J., Prieur, C., Coron, J.-M., d'Andréa Novel, B., \& Bastin, G. (2003). Boundary feedback control in networks of open channels. Automatica, 39(8), 1365-1376.

Diagne, A., Bastin, G., \& Coron, J.-M. (2012). Lyapunov exponential stability of 1-d linear hyperbolic systems of balance laws. Automatica, 48(1), 109-114.

Diagne, A., Diagne, M., Tang, S., \& Krstic, M. (2017). Backstepping stabilization of the linearized Saint-Venant-Exner model. Automatica, 76, 345 - 354.

Diagne, M., Tang, S.-X., Diagne, A., \& Krstic, M. (2017). Control of shallow waves of two unmixed fluids by backstepping. Ann. Rev. in Control, 44, $211-225$.

Dick, M., Gugat, M., Herty, M., Leugering, G., Steffensen, S., \& Wang, K. (2014). Stabilization of networked hyperbolic systems with boundary feedback. In Trends in PDE constrained optimization (pp. 487-504). Springer.

Di Meglio, F., Vazquez, R., \& Krstic, M. (2013). Stabilization of a system of $n+1$ coupled first-order hyperbolic linear PDEs with a single boundary input. IEEE Transactions on Automatic Control, 12(58), 3097-3111.

Dos Santos, V., Bastin, G., Coron, J.-M., \& d'Andréa Novel, B. (2008). Boundary control with integral action for hyperbolic systems of conservation laws: stability and experiments. Automatica, 44(5), 1310-1318.

Gottlich, S., \& Schillen, P. (2017). Numerical discretization of boundary control problems for systems of balance laws: Feedback stabilisation. European Journal of Control, 35, 11-18.

Gugat, M., \& Herty, M. (2011). Existence of classical solutions and feedback stabilization for the flow in gas networks. ESAIM: Control, Optimisation and Calculus of Variations, $17(01), 28-51$.

Gugat, M., Herty, M., Klar, A., Leugering, G., \& Schleper, V. (2012). Well-posedness of networked hyperbolic systems of balance laws. In Constrained optimization and optimal control for partial differential equations (pp. 123-146). Springer.

Hudson, J., \& Sweby, P. K. (2003). Formulations for numerically approximating hyperbolic systems governing sediment transport. Journal of Scientific Computing, 19(1-3), 225-252.

Karaguler, T. (2008). A new method for solving transient Lossy transmission line problem. In Numerical analysis and its applications (pp. 338-344). Springer.

LeVeque, R. J. (2002). Finite volume methods for hyperbolic problems (Vol. 31). Cambridge: Cambridge University Press.

Litrico, X., \& Fromion, V. (2009). Boundary control of hyperbolic conservation laws using a frequency domain approach. Automatica, 45(3), 647-656.

Prieur, C. (2009). Control of systems of conservation laws with boundary errors. NHM, $4(2)$, 393-407.

Prieur, C., Girard, A., \& Witrant, E. (2012). Lyapunov functions for switched linear hyperbolic systems. In 4th IFAC conference on analysis and design of hybrid systems (adhs'12).

Prieur, C., Girard, A., \& Witrant, E. (2014). Stability of switched linear hyperbolic systems by Lyapunov techniques. Automatic Control, IEEE Transactions on, 59(8), 2196-2202.

Prieur, C., Winkin, J., \& Bastin, G. (2008). Robust boundary control of systems of conservation laws. Mathematics of Control, Signals, and Systems, 20(2), 173-197.

Siebel, F., \& Mauser, W. (2006). On the fundamental diagram of traffic flow. SIAM Journal on Applied Mathematics, 66(4), 1150-1162.

Vazquez, R., Coron, J.-M., Krstic, M., \& Bastin, G. (2011). Local exponential $H^{2}$ stabilization of a $2 \times 2$ quasilinear hyperbolic system using backstepping. In Decision and Control and European Control Conference (CDC-ECC), 2011 50th IEEE conference on (pp. 1329-1334).

Weldegiyorgis, G. (2017). Numerical stabilization with boundary controls for hyperbolic systems of balance laws. (Preprint on webpage at http://hdl.handle.net/2263/60870, Accessed: 2017 Aug 19) 


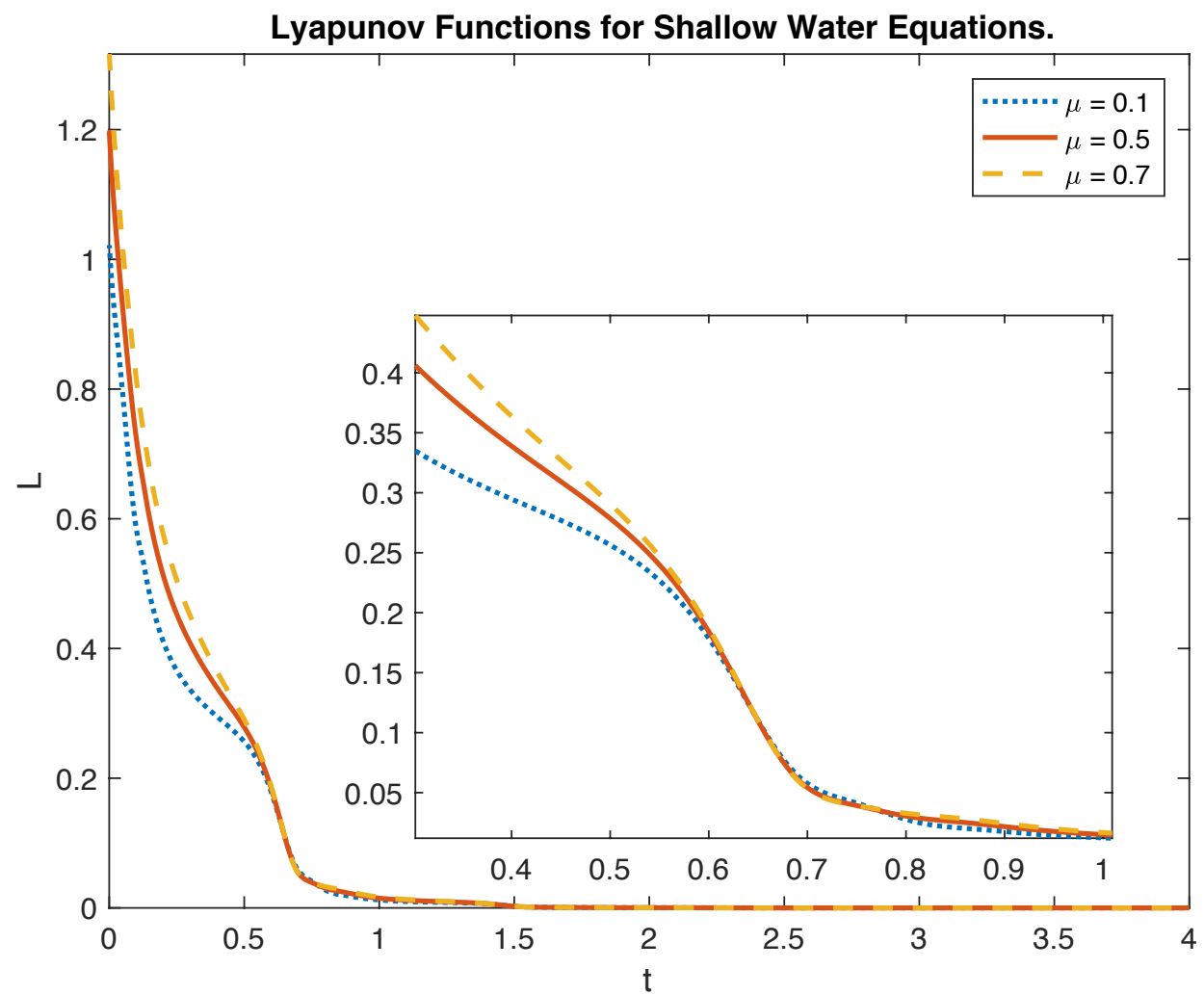

Figure 1. The decay of Lyapunov function for Saint-Venant equations. The choice of parameters are $p_{1}=$ $0.0992, p_{2}=0.2008, k_{12}=0.3$ and $k_{21}=0.8$ with $l=1, J=200$ and $T=10$ under CFL $=0.75$. 


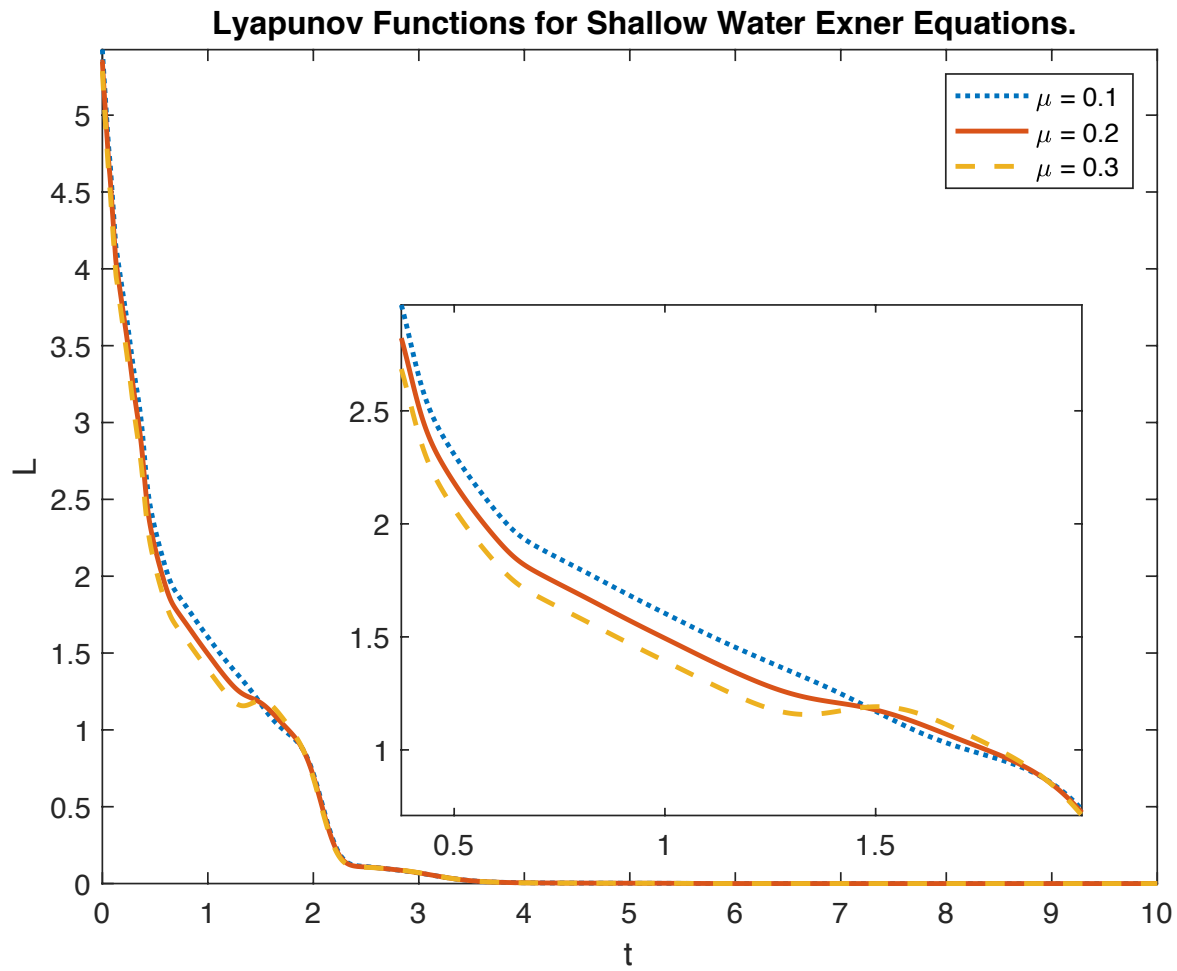

Figure 2. The decay of Lyapunov function for Saint-Venant-Exner equations. The choice of parameters are $p_{1}=0.1014, p_{2}=0.0267, p_{3}=0.1719, k_{13}=0.6, k_{23}=0.5, k_{31}=0.15$ and $k_{32}=0.15$, with $l=1, J=200$ and $T=4$ under $\mathrm{CFL}=0.75$. 